\title{
GROUP ACTIONS ON TOPOLOGICAL GRAPHS
}

\author{
VALENTIN DEACONU, ALEX KUMJIAN, AND JOHN QUIGG
}

\begin{abstract}
We define the action of a locally compact group $G$ on a topological graph $E$. This action induces a natural action of $G$ on the $C^{*}$-correspondence $\mathcal{H}(E)$ and on the graph $C^{*}$-algebra $C^{*}(E)$. If the action is free and proper, we prove that $C^{*}(E) \rtimes_{r} G$ is strongly Morita equivalent to $C^{*}(E / G)$. We define the skew product of a locally compact group $G$ by a topological graph $E$ via a cocycle $c: E^{1} \rightarrow G$. The group acts freely and properly on this new topological graph $E \times{ }_{c} G$. If $G$ is abelian, there is a dual action on $C^{*}(E)$ such that $C^{*}(E) \rtimes \hat{G} \cong C^{*}\left(E \times{ }_{c} G\right)$. We also define the fundamental group and the universal covering of a topological graph.
\end{abstract}

\section{INTRODUCTION}

Topological graphs generalize discrete directed graphs. They have a richer structure, since the vertex and edge spaces may be arbitrary locally compact spaces, and it is natural to study their symmetries. The corresponding $C^{*}$-algebras provide a wealth of intriguing examples. Katsura proved in Kat08 that all Kirchberg algebras can be obtained from topological graphs.

In [KP99] the authors consider free actions of discrete groups $G$ on directed graphs $E$ and on their associated $C^{*}$-algebras $C^{*}(E)$. They prove that the crossed product $C^{*}(E) \rtimes G$ is strongly Morita equivalent to $C^{*}(E / G)$, where $E / G$ is the quotient graph. They also consider the notion of skew product graph associated to a cocycle $c: E^{1} \rightarrow G$ and the universal covering tree of a graph.

In this paper, we prove an analogous result for the reduced crossed product for $G$ a locally compact group acting freely and properly on a topological graph $E$. Unlike [KP99, where groupoids were used extensively, the $C^{*}$-algebra $C^{*}(E)$ is defined here using a $C^{*}$ - correspondence $\mathcal{H}(E)$ and the Cuntz-Pimsner construction (see Kat04). The main ingredients of the proof are a structure theorem of Palais about principal $G$-bundles, the generalized fixed point algebras of Rieffel, and the use of multiplier bimodules introduced by Echterhoff, Raeburn, and others. We need multiplier bimodules because we must construct a homomorphism of $C^{*}(E / G)$ into the multiplier algebra $M\left(C^{*}(E)\right)$, and as an intermediate step we map the correspondence $\mathcal{H}(E / G)$ into the multipliers of the correspondence $\mathcal{H}(E)$.

We also define the notion of fundamental group of a topological graph and the universal covering, using a space $R(E)$, called the geometric realization, which is a kind of double mapping torus. In the discrete case, the geometric realization is the usual 1-dimensional CW-complex that we associate with a graph, and the fundamental group is free. For a topological graph $E$, the space $R(E)$ could be a

Date: October 22, 2018.

1991 Mathematics Subject Classification. Primary 46L05; Secondary 46L55.

Key words and phrases. $C^{*}$-algebra, group action, topological graph. 
higher dimensional CW-complex, and $\pi_{1}(E)$ is not necessarily free, as we will see in our examples.

Our paper is organized as follows. In section 2 we recall the notion of topological graph and the construction of its $C^{*}$-algebra as a Cuntz-Pimsner algebra, giving several examples. In section 3 we define group actions on topological graphs, and prove a structure theorem about topological graphs on which a locally compact group acts freely and properly. The fundamental group of a topological graph and the corresponding universal covering are defined in section 4; we also consider a number of examples. In section 5 we discuss group actions on $C^{*}$-correspondences, recall the notion of generalized fixed point algebra, and prove the main theorem stating that $C^{*}(E) \rtimes_{r} G$ and $C^{*}(E / G)$ are strongly Morita equivalent for a free and proper action. The appendix contains basic results concerning multipliers of correspondences which are needed in section 5 .

The authors would like to thank the referee for a number of helpful comments.

\section{TOPOlOGical GRAPHS AND THEIR $C^{*}$-ALGEBRAS}

Let $E=\left(E^{0}, E^{1}, s, r\right)$ be a topological graph. Recall that $E^{0}$ and $E^{1}$ are locally compact (Hausdorff) spaces, and that $s, r: E^{1} \rightarrow E^{0}$ are continuous maps such that $s$ is a local homeomorphism. We think of points in $E^{0}$ as vertices, and of points $e \in E^{1}$ as edges from $s(e)$ to $r(e)$. A path of length $n$ in $E=\left(E^{0}, E^{1}, s, r\right)$ is a sequence $e_{1} e_{2} \cdots e_{n}$ of edges such that $s\left(e_{i}\right)=r\left(e_{i+1}\right)$ for all $i$. We denote by $E^{n}$ the set of paths of length $n$. The space of infinite paths is denoted $E^{\infty}$. The maps $s$ and $r$ extend naturally to $E^{n}$, and $\left(E^{0}, E^{n}, s, r\right)$ becomes a topological graph. A vertex $v \in E^{0}$ is viewed as a path of length 0 .

We define two open subsets $E_{\mathrm{sce}}^{0}, E_{\text {fin }}^{0}$ of $E^{0}$ by

$$
\begin{gathered}
E_{\mathrm{sce}}^{0}=\left\{v \in E^{0}: v \text { has a neighborhood } V \text { such that } r^{-1}(V)=\emptyset\right\}, \\
E_{\text {fin }}^{0}=\left\{v \in E^{0}: v \text { has a neighborhood } V \text { such that } r^{-1}(V) \text { is compact }\right\},
\end{gathered}
$$

and the set of regular vertices by $E_{\mathrm{rg}}^{0}:=E_{\mathrm{fin}}^{0} \backslash \overline{E_{\mathrm{sce}}^{0}}$. It is proved in Proposition 2.8 of Kat04 that $v \in E_{\mathrm{rg}}^{0}$ if and only if $v$ has a neighborhood such that $r^{-1}(V)$ is compact and $r\left(r^{-1}(V)\right)=V$. In particular, if $r$ is proper and surjective, we have $E_{\mathrm{rg}}^{0}=E^{0}$.

The $C^{*}$-algebra $C^{*}(E)$ of a topological graph is defined as the Cuntz-Pimsner algebra $\mathcal{O}_{\mathcal{H}}$ of the $C^{*}$-correspondence $\mathcal{H}=\mathcal{H}(E)$ over the $C^{*}$-algebra $A=C_{0}\left(E^{0}\right)$, which is obtained as a completion of $C_{c}\left(E^{1}\right)$ using the inner product

$$
\langle\xi, \eta\rangle(v)=\sum_{s(e)=v} \overline{\xi(e)} \eta(e), \xi, \eta \in C_{c}\left(E^{1}\right)
$$

and the multiplications

$$
(\xi \cdot f)(e)=\xi(e) f(s(e)),(f \cdot \xi)(e)=f(r(e)) \xi(e) .
$$

The Hilbert $C_{0}\left(E^{0}\right)$-module $\mathcal{H}$ can be identified with $\left\{\xi \in C_{0}\left(E^{1}\right) \mid\langle\xi, \xi\rangle \in\right.$ $\left.C_{0}\left(E^{0}\right)\right\}$. There exists an injective $*$-homomorphism $\pi_{E}: C_{b}\left(E^{1}\right) \rightarrow \mathcal{L}(\mathcal{H})$ given by $\left(\pi_{E}(f) \xi\right)(e)=f(e) \xi(e)$ such that $\pi_{E}(f) \in \mathcal{K}(\mathcal{H})$ if and only if $f \in C_{0}\left(E^{1}\right)$.

For more details, see Kat04.

Recall that a Toeplitz representation of a $C^{*}$-correspondence $\mathcal{H}$ over $A$ in a $C^{*}$-algebra $C$ is a pair $(\tau, \pi)$ with $\tau: \mathcal{H} \rightarrow C$ a linear map and $\pi: A \rightarrow C$ a 
*-homomorphism, such that

$$
\tau(\xi a)=\tau(\xi) \pi(a), \tau(\xi)^{*} \tau(\eta)=\pi(\langle\xi, \eta\rangle), \tau(\varphi(a) \xi)=\pi(a) \tau(\xi),
$$

where $\varphi: A \rightarrow \mathcal{L}(\mathcal{H})$ is the left multiplication. The corresponding universal $C^{*}$ algebra is called the Toeplitz algebra $\mathcal{T}_{\mathcal{H}}$. A representation $(\tau, \pi)$ is covariant if $\pi(a)=\tau^{(1)}(\varphi(a))$ for all $a$ in the ideal $\varphi^{-1}(\mathcal{K}(\mathcal{H})) \cap(\operatorname{ker} \varphi)^{\perp}$, where $\tau^{(1)}: \mathcal{K}(\mathcal{H}) \rightarrow C$ is such that $\tau^{(1)}\left(\theta_{\xi, \eta}\right)=\tau(\xi) \tau(\eta)^{*}$. The Cuntz-Pimsner algebra $\mathcal{O}_{\mathcal{H}}$ is universal with respect to covariant representations, and it is a quotient of $\mathcal{T}_{\mathcal{H}}$. Let $\left(k_{\mathcal{H}}, k_{A}\right)$ denote the canonical Toeplitz representation of the $A$-correspondence $\mathcal{H}$ in $\mathcal{O}_{\mathcal{H}}$. A covariant representation $(\tau, \pi)$ of $\mathcal{H}$ in a $C^{*}$-algebra $C$ gives rise to a $*$-homomorphism $\tau \times \pi$ : $\mathcal{O}_{\mathcal{H}} \rightarrow C$ such that $\tau=(\tau \times \pi) \circ k_{\mathcal{H}}$ and $\pi=(\tau \times \pi) \circ k_{A}$.

Remark 2.1. In the case of a $C^{*}$-correspondence $\mathcal{H}(E)$ associated with a topological graph as above, it is proved in Proposition 1.24 of Kat04 that $\operatorname{ker} \varphi=C_{0}\left(E_{\text {sce }}^{0}\right)$ and that $\varphi^{-1}(\mathcal{K}(H))=C_{0}\left(E_{\text {fin }}^{0}\right)$, therefore the ideal $\varphi^{-1}(\mathcal{K}(\mathcal{H})) \cap(\operatorname{ker} \varphi)^{\perp}$ coincides with $C_{0}\left(E_{\mathrm{rg}}^{0}\right)$.

Example 2.2. If the vertex space $E^{0}$ is discrete, then the edge space $E^{1}$ is also discrete, and $E=\left(E^{0}, E^{1}, s, r\right)$ is an usual (discrete) graph. For a discrete graph $E$, its $C^{*}$-algebra $C^{*}(E)$ was initially defined as the universal $C^{*}$-algebra generated by mutually orthogonal projections $p_{v}$ for $v \in E^{0}$ and partial isometries $t_{e}$ for $e \in E^{1}$ with orthogonal ranges such that $t_{e}^{*} t_{e}=p_{s(e)}, t_{e} t_{e}^{*} \leq p_{r(e)}$ and

$$
p_{v}=\sum_{r(e)=v} t_{e} t_{e}^{*} \text { if } 0<\left|r^{-1}(v)\right|<\infty
$$

If $\mathcal{H}=\mathcal{H}(E)$ is the associated $C^{*}$-correspondence described above, then

$$
\begin{gathered}
\varphi^{-1}(\mathcal{K}(H))=C_{0}\left(\left\{v \in E^{0}:\left|r^{-1}(v)\right|<\infty\right\}\right), \\
\operatorname{ker}(\varphi)=C_{0}\left(\left\{v \in E^{0}:\left|r^{-1}(v)\right|=0\right\}\right),
\end{gathered}
$$

since $E_{\mathrm{fin}}^{0}=\left\{v \in E^{0}: r^{-1}(v)\right.$ is finite $\}$ and $E_{\mathrm{sce}}^{0}=\left\{v \in E^{0}: r^{-1}(v)=\emptyset\right\}$ is the set of sources. The maps

$$
\pi(f)=\sum_{v \in E^{0}} f(v) p_{v}, f \in C_{0}\left(E^{0}\right), \quad \tau(\xi)=\sum_{e \in E^{1}} \xi(e) t_{e}, \xi \in C_{c}\left(E^{1}\right)
$$

give a Toeplitz representation of $\mathcal{H}$ into $C^{*}(E)$ if and only if $t_{e}^{*} t_{e}=p_{s(e)}$ and $t_{e} t_{e}^{*} \leq p_{r(e)}$, which is covariant if and only if $(1)$ is satisfied. Hence, the $C^{*}$-algebra of a discrete graph $E$, defined using generators and relations, is isomorphic to the Cuntz-Pimsner algebra $\mathcal{O}_{\mathcal{H}}$ (see [FLR00, Proposition 12]) and [Kat04, Example 1]).

Example 2.3. Let $E^{0}=E^{1}=\mathbb{T}, s(z)=z$, and $r(z)=e^{2 \pi i \theta} z$ for $\theta \in[0,1]$ irrational. Then $C^{*}(E) \cong A_{\theta}$, the irrational rotation algebra. More generally, let $E^{0}=E^{1}=X$, where $X$ is a locally compact metric space, let $s=$ id and let $r=h$ for $h: X \rightarrow X$ a homeomorphism. Then $C^{*}(E) \cong C_{0}(X) \rtimes \mathbb{Z}$, since $C^{*}(E)$ is the universal $C^{*}$-algebra generated by a copy of $C_{0}(X)$ and a unitary $u$ satisfying $\hat{h}(f)=u^{*} f u$ for $f \in C_{0}(X)$, where $\hat{h}(f)=f \circ h$.

Example 2.4. Let $n \in \mathbb{N} \backslash\{0\}$ and let $m \in \mathbb{Z} \backslash\{0\}$. Take $E^{0}=E^{1}=\mathbb{T}, s(z)=$ $z^{n}, r(z)=z^{m}$. We get a topological graph with both $s$ and $r$ local homeomorphisms. When $m \notin n \mathbb{Z}, C^{*}(E)$ is simple and purely infinite, see [Kat08, Example A.6]. 
Example 2.5. (The Cayley graph of a finitely generated locally compact group). A locally compact group $G$ is called finitely generated if there is a finite subset $S$ of $G$ such that the closure of the subgroup generated by $S$ is all $G$. Given a set of generators $S=\left\{h_{1}, h_{2}, \ldots, h_{n}\right\}$, define the associated Cayley graph $E=E(G, S)$ with $E^{0}=G, E^{1}=S \times G, s(h, g)=g$, and $r(h, g)=g h$. Note that $E(G, S)$ becomes a topological graph and that both $s$ and $r$ are local homeomorphisms. For $G$ discrete and finitely generated, we recover the usual notion of Cayley graph. The Cayley graph may change if we change the set of generators.

For $G=(\mathbb{R},+)$ and $S=\{1, \theta\}$, where $\theta$ is irrational, the corresponding Cayley graph $E$ has $E^{0}=\mathbb{R}, E^{1}=\{1, \theta\} \times \mathbb{R}, s(1, x)=x, r(1, x)=x+1, s(\theta, x)=x$, and $r(\theta, x)=x+\theta$. Its $C^{*}$-algebra is simple and, if $\theta<0$, it is purely infinite; moreover, $C^{*}(E) \cong \mathcal{O}_{2} \rtimes_{\alpha} \mathbb{R}$, where $\alpha_{t}\left(S_{1}\right)=e^{i t} S_{1}, \alpha_{t}\left(S_{2}\right)=e^{i t \theta} S_{2}$ for $t \in \mathbb{R}$ and for the standard generators $S_{1}, S_{2}$ of the Cuntz algebra $\mathcal{O}_{2}$ (see Theorem 2, section 3 in KK97] and Kat02, Proposition 4.3]).

The above example should be regarded as a special case of the following.

Example 2.6. (Skew products of topological graphs). Let $E=\left(E^{0}, E^{1}, s, r\right)$ be a topological graph, let $G$ be a locally compact group, and let $c: E^{1} \rightarrow G$ be a continuous function. We call $c$ a cocycle, and define the skew product graph $E \times{ }_{c} G=\left(E^{0} \times G, E^{1} \times G, \tilde{s}, \tilde{r}\right)($ cf. [KP99, Rae05] $)$, where

$$
\tilde{s}(e, g)=(s(e), g), \quad \tilde{r}(e, g)=(r(e), g c(e)) .
$$

Notice that $E \times{ }_{c} G$ becomes a topological graph using the product topology, since $\tilde{s}$ is a local homeomorphism and $\tilde{r}$ is continuous. The situation of a graph with one vertex and $n$ loops $\left\{e_{1}, \ldots, e_{n}\right\}$ and a set of generators $S=\left\{h_{1}, \ldots, h_{n}\right\}$ of a group $G$ such that $c\left(e_{i}\right)=h_{i}, i=1, \ldots, n$ gives the Cayley graph $E(G, S)$ as a skew product.

In particular, if $E$ is the graph with one vertex and two edges $\{e, f\}$ and $G=$ $(\mathbb{R},+)$, the map $c:\{e, f\} \rightarrow \mathbb{R}, c(e)=1, c(f)=\theta$ for $\theta$ irrational determines the topological graph described in the above example.

If $E$ is an arbitrary discrete graph and $G=\mathbb{Z}$ with $c(e)=1$ for all $e \in E^{1}$, then $E \times{ }_{c} G$ is the product graph $E \times Z$, where $Z^{0}=Z^{1}=\mathbb{Z}$, with $s(k)=k, r(k)=k+1$. There is an isomorphism $C^{*}\left(E \times_{c} G\right) \cong C^{*}(E) \rtimes \mathbb{T}$ (see [KP99, Proposition 2.6]).

\section{Group aCtions on topologicAl GRAPHS}

Definition 3.1. Let $E, F$ be two topological graphs. A graph morphism $\varphi: E \rightarrow F$ is a pair of continuous maps $\varphi=\left(\varphi^{0}, \varphi^{1}\right)$ where $\varphi^{i}: E^{i} \rightarrow F^{i}, i=0,1$ such that $\varphi^{0} \circ r=r \circ \varphi^{1}$ and $\varphi^{0} \circ s=s \circ \varphi^{1}$, i.e. the diagram

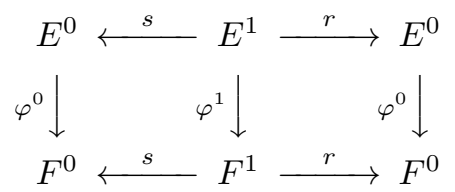

is commutative.

A graph morphism has the unique path lifting property for $s$ if $\varphi^{0}, \varphi^{1}$ are surjective and for every $v \in E^{0}$ and every $f \in F^{1}$ with $s(f)=\varphi^{0}(v)$ there is a unique $e \in E^{1}$ such that $\varphi^{1}(e)=f$ and $s(e)=v$. A graph morphism $\varphi$ is a graph covering if both $\varphi^{0}, \varphi^{1}$ are covering maps. In that case, $\varphi$ has the unique path lifting property with respect to both $s$ and $r$. 
An isomorphism of topological graphs is a graph morphism $\varphi=\left(\varphi^{0}, \varphi^{1}\right)$ such that $\varphi^{i}$ is a homeomorphism for $i=0,1$. It follows that $\varphi^{-1}=\left(\left(\varphi^{0}\right)^{-1},\left(\varphi^{1}\right)^{-1}\right)$ is also a graph morphism. We denote by $\operatorname{Aut}(E)$ the group of automorphisms of a topological graph $E$.

A locally compact group $G$ acts on $E$ if there are continuous maps $\lambda^{i}: G \times E^{i} \rightarrow$ $E^{i}$, and we write $\lambda^{i}(g, x)=\lambda_{g}^{i}(x)$ for $i=0,1$, such that $g \mapsto \lambda_{g}$ is a group homomorphism from $G$ into $\operatorname{Aut}(E)$. The action $\lambda$ is called free if $\lambda_{g}^{0}(v)=v$ for some $v \in E^{0}$ implies $g=1_{G}$. Note that in this case the action of $G$ is also free on the edges of $E$. The action is called proper if the maps $G \times E^{0} \rightarrow E^{0} \times E^{0},(g, v) \mapsto\left(\lambda_{g}^{0}(v), v\right)$ and $G \times E^{1} \rightarrow E^{1} \times E^{1},(g, e) \mapsto\left(\lambda_{g}^{1}(e), e\right)$ are proper. In fact, by Proposition 2.1.14 in ADR00, it is sufficient to require properness of the first map. We will frequently write $g \cdot e$ for $\lambda_{g}^{1}(e)$, and similarly for the action on vertices.

Definition 3.2. Let $P, X$ be locally compact Hausdorff spaces, and let $G$ be a locally compact group. A map $P \stackrel{q}{\longrightarrow} X$ is called a principal $G$-bundle if there is a free and proper action of $G$ on $P$ and $q$ induces an identification of $P / G$ with $X$.

Note that we make no assumption regarding local triviality. Principal bundles are thoroughly discussed in Pal61 and Hus94 using various definitions. By [RW98, Remark 4.64] the notion of principal G-bundle in [Hus94] is equivalent to our notion determined by a free and proper action.

Given a principal $G$-bundle $P \stackrel{q}{\longrightarrow} X$ and a continuous function $f: Y \rightarrow X$, we may view the pull-back $f^{*}(P)=\{(y, p) \in Y \times P: f(y)=q(p)\}$ as a principal $G$-bundle $f^{*}(P) \stackrel{\pi_{1}}{\longrightarrow} Y$ where the action of $G$ is given by $g \cdot(y, p)=(y, g \cdot p)$.

The following result may be found in Hus94, Theorem 4.4.2] (see also the discussion preceding [Pal61, Proposition 1.3.4]).

Lemma 3.3. Suppose we are given principal $G$-bundles $P_{i} \stackrel{q_{i}}{\longrightarrow} X_{i}$ for $i=1,2$ and a pair of continuous maps $f: X_{1} \rightarrow X_{2}, \tilde{f}: P_{1} \rightarrow P_{2}$ such that $\tilde{f}$ is equivariant and $q_{2} \tilde{f}=f q_{1}$. Then $\theta_{f}: P_{1} \rightarrow f^{*}\left(P_{2}\right)$ given by $\theta_{f}(p)=\left(q_{1}(p), \tilde{f}(p)\right)$ is an isomorphism of G-bundles.

The following is a structure theorem for topological graphs equipped with a free and proper action of a locally compact group: the quotient is a topological graph, and moreover the original graph can be reconstructed from the quotient graph and certain additional data.

Theorem 3.4. Let $\left(F^{0}, F^{1}, s, r\right)$ be a topological graph and $G$ be a locally compact group. Given a principal $G$-bundle $P \stackrel{q}{\longrightarrow} F^{0}$ and an isomorphism of pull-backs $\theta: s^{*}(P) \cong r^{*}(P)$, we may construct a topological graph $\left(E^{0}, E^{1}, \tilde{s}, \tilde{r}\right)$ with a free and proper action of $G$ by setting $E^{0}:=P$ and $E^{1}:=s^{*}(P)$ (with structure maps defined in the obvious way). Conversely, every topological graph $\left(E^{0}, E^{1}, \tilde{s}, \tilde{r}\right)$ on which $G$ acts freely and properly is isomorphic to one arising in this way.

Proof. First suppose we are given $P, q$, and $\theta$. Define $\tilde{s}: s^{*}(P) \rightarrow P$ by $\tilde{s}=\pi_{2}$, i.e., $\tilde{s}(e, v)=v$, and $\tilde{r}: s^{*}(P) \rightarrow P$ by $\tilde{r}=\pi_{2} \circ \theta$. To see that $\tilde{s}$ is a local homeomorphism, fix a point in $\tilde{e}=(e, v) \in s^{*}(P)$. Since $s$ is a local homeomorphism, there is an open set $U \subset F^{1}$ containing $e$ such that the restriction $\left.s\right|_{U}$ is injective. It follows that the restriction $\left.\tilde{s}\right|_{\pi_{1}^{-1}(U)}$ is also injective, and hence that $\tilde{s}$ is a local homeomorphism. 
The following commutative diagram illustrates the above construction:

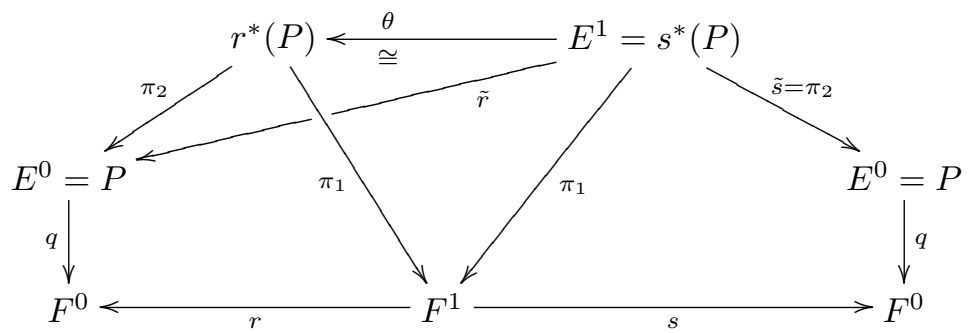

Since $\pi_{1} \circ \theta=\pi_{1}, \theta$ has the following form:

$$
\theta(e, v)=(e, \tilde{r}(e, v)) \text { for } \quad(e, v) \in s^{*}(P) .
$$

The converse essentially follows from Lemma 3.3 above; the only non-obvious bit is showing that the quotient is a topological graph. So, suppose $G$ acts freely and properly on a topological graph $E=\left(E^{0}, E^{1}, \tilde{s}, \tilde{r}\right)$. Let $F^{i}=E^{i} / G$ be the quotient spaces, with quotient maps $q^{i}: E^{i} \rightarrow F^{i}$, for $i=0,1$. Since the $G$-action on $E$ is compatible with the range and source maps, we have the following diagram of principal $G$-bundles:

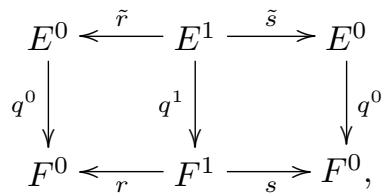

where $r, s: F^{1} \rightarrow F^{0}$ are continuous, and $s$ is open. We must show that $F=$ $\left(F^{0}, F^{1}, s, r\right)$ is a topological graph, and that there is an isomorphism of pull-backs $\theta: s^{*}\left(E^{0}\right) \rightarrow r^{*}\left(E^{0}\right)$ such that $E$ is isomorphic to the topological graph with the same vertex space $E^{0}$, but with edge space $s^{*}\left(E^{0}\right)$, source map $\pi_{2}$, and range map $\pi_{2} \circ \theta$. By Lemma 3.3 there are $G$-bundle isomorphisms $\theta_{r}, \theta_{s}$ making the following diagram commute (without the top arrow $\theta$ ):

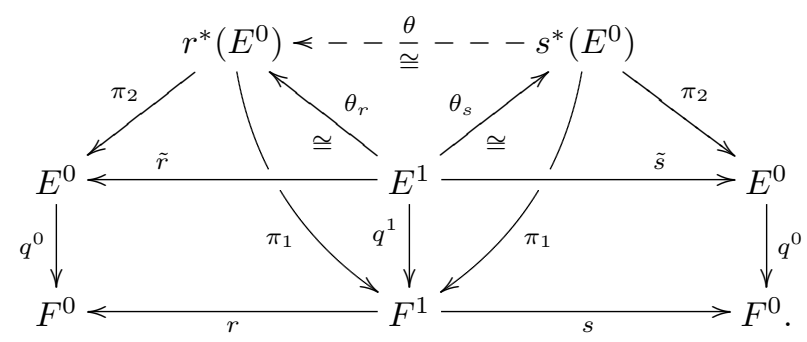

Of course we define the $G$-bundle isomorphism $\theta$ so that the top triangle (and hence the entire diagram) commutes.

It only remains to show that $s: F^{1} \rightarrow F^{0}$ is locally injective. Let $e \in F^{1}$, and put $v=s(e)$. Fix $u \in E^{0}$ with $q^{0}(u)=v$, so that $(e, u) \in s^{*}\left(E^{0}\right)$. Since $E$ is a topological graph, we can find open neighborhoods $U$ of $e$ in $F^{1}$ and $V$ of $u$ in $E^{0}$ such that $\pi_{2}: s^{*}\left(E^{0}\right) \rightarrow E^{0}$ is injective on

$$
(U \times V) \cap s^{*}\left(E^{0}\right) .
$$

Since $q^{0}: E^{0} \rightarrow F^{0}$ is open, we can shrink $U$ if necessary so that $s(U) \subset q^{0}(V)$. 
Let $e_{1}, e_{2} \in U$, and assume that

$$
s\left(e_{1}\right)=s\left(e_{2}\right)=v^{\prime} .
$$

Choose $u^{\prime} \in V$ such that $q^{0}\left(u^{\prime}\right)=v^{\prime}$. Then $\left(e_{1}, u^{\prime}\right),\left(e_{2}, u^{\prime}\right) \in(U \times V) \cap s^{*}\left(E^{0}\right)$, so we must have $e_{1}=e_{2}$.

Example 3.5. If we are given a finitely generated locally compact group $G$ with generating set $S$, then $G$ acts freely and properly on its Cayley graph $E=E(G, S)$ by $\lambda_{g}^{0}\left(g^{\prime}\right)=g g^{\prime}$ and $\lambda_{g}^{1}\left(h, g^{\prime}\right)=\left(h, g g^{\prime}\right)$. The quotient graph $E / G$ has $|S|$ loops and one vertex.

Example 3.6. If $E=\left(E^{0}, E^{1}, s, r\right)$ is a topological graph, $G$ is a locally compact group, and $c: E^{1} \rightarrow G$ is a continuous function, then $\varphi: E \times{ }_{c} G \rightarrow E, \varphi(g, x)=$ $x$ is a principal $G$-bundle map. Indeed, $G$ acts freely and properly on $E \times{ }_{c} G$ by $\lambda_{g}^{0}(v, h)=(v, g h)$ and $\lambda_{g}^{1}(e, h)=(e, g h)$. The source and range maps are equivariant, and the quotient graph is isomorphic to $E$.

The following result characterizes skew products:

Corollary 3.7. The topological graph constructed in Theorem 3.4 from an isomorphism $\theta: s^{*}(P) \cong r^{*}(P)$ is $G$-equivariantly isomorphic to a skew product $F \times{ }_{c} G$ if and only if the $G$-bundle $P$ over $F^{0}$ is trivial.

Proof. The forward direction is trivial, so assume that the $G$-bundle $q: P \rightarrow F^{0}$ is trivial, and let $E$ be the topological graph constructed from an isomorphism $\theta: s^{*}(P) \rightarrow r^{*}(P)$. The pull-back of a trivial bundle is trivial, so most of what we have to do is quite straightforward; the only slightly non-obvious bit is that the isomorphism $s^{*}(P) \cong F^{1} \times G$ of $G$-bundles can be promoted to a topological-graph isomorphism $E \cong F \times{ }_{c} G$ for a suitable cocycle $c$.

Let $\varphi^{0}: P \rightarrow F^{0} \times G$ be a $G$-bundle isomorphism. We must show that there are:

- a $G$-bundle isomorphism $\varphi^{1}: E^{1} \rightarrow F^{1} \times G$, and

- a cocycle $c: F^{1} \rightarrow G$

such that $\varphi=\left(\varphi^{0}, \varphi^{1}\right): E \rightarrow F \times{ }_{c} G$ is an isomorphism of topological graphs.

The diagram

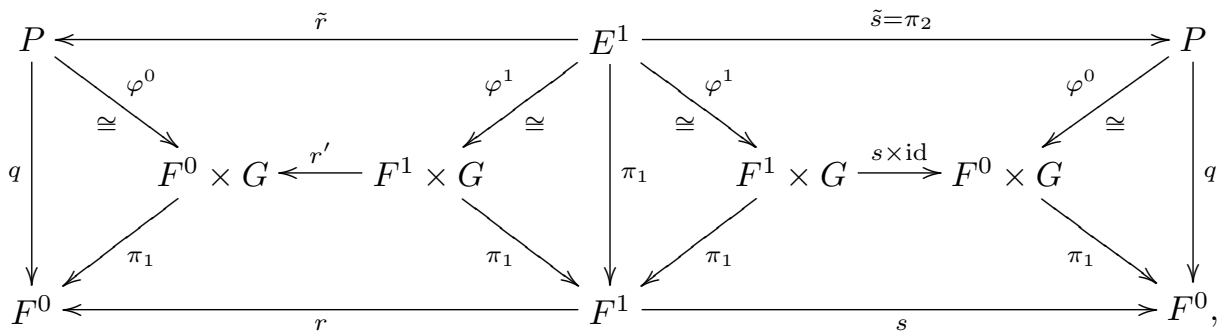

where

$$
r^{\prime}:\left(F \times{ }_{c} G\right)^{1}=F^{1} \times G \rightarrow F^{0} \times G=\left(F \times{ }_{c} G\right)^{0}
$$

is the range map for the skew product, given by

$$
r^{\prime}(e, g)=(r(e), g c(e)),
$$

illustrates the hypothesis and conclusion. Keep in mind that $E^{0}=P$ and $E^{1}=$ $s^{*}(P)$. We are given the map $\varphi^{0}$, and since it is a $G$-bundle isomorphism the extreme left and right triangles commute. 
It is an elementary fact about pull-backs that there is a $G$-bundle isomorphism $\varphi^{1}: s^{*}(P) \rightarrow F^{1} \times G$ making the right half of the diagram (2) commute. We can now define $r^{\prime}: F^{1} \times G \rightarrow F^{0} \times G$ so that the left half of (2) commutes. It remains to show that there is a continuous map $c: F^{1} \rightarrow G$ such that $r^{\prime}$ has the form

$$
r^{\prime}(e, g)=(r(e), g c(e)) \text {. }
$$

Since

$$
\pi_{1} \circ r^{\prime}=r \circ \pi_{1}: F^{1} \times G \rightarrow F^{0},
$$

we have $\pi_{1}\left(r^{\prime}(e, 1)\right)=r(e)$. Define $c: F^{1} \rightarrow G$ by

$$
c(e)=\pi_{2}\left(r^{\prime}(e, 1)\right)
$$

Then $c$ is continuous, and we have

$$
\begin{aligned}
r^{\prime}(e, g) & =r^{\prime}(g \cdot(e, 1)) \\
& =g \cdot r^{\prime}(e, 1) \\
& =g \cdot(r(e), c(e)) \\
& =(r(e), g c(e)) .
\end{aligned}
$$

Lemma 3.8. If we are given principal $G$-bundles $P_{i} \stackrel{q_{i}}{\longrightarrow} X_{i}$ for $i=1,2$ and a pair of continuous maps $f: X_{1} \rightarrow X_{2}, \tilde{f}: P_{1} \rightarrow P_{2}$ such that $\tilde{f}$ is equivariant and $q_{2} \tilde{f}=f q_{1}$, and compact sets $K \subset X_{1}$ and $L \subset P_{2}$, then $\left(q_{1}\right)^{-1}(K) \cap \tilde{f}^{-1}(L)$ is a compact subset of $P_{1}$.

Proof. Observe that $f^{*}\left(P_{2}\right)$ is a closed subset of $X_{1} \times P_{2}$ and hence $(K \times L) \cap f^{*}\left(P_{2}\right)$ is compact. Therefore,

$$
q_{1}^{-1}(K) \cap \tilde{f}^{-1}(L)=\left(\theta_{f}\right)^{-1}\left((K \times L) \cap f^{*}\left(P_{2}\right)\right)
$$

is also compact.

Corollary 3.9. Let a locally compact group $G$ act freely and properly on a topological graph $E=\left(E^{0}, E^{1}, s, r\right)$, let $q_{1}: E^{1} \rightarrow E^{1} / G$ be the quotient map, and let $K \subset E^{1} / G$ be compact. Then:

(1) For every compact subset $L \subset E^{0}$, both intersections

$$
q_{1}^{-1}(K) \cap r^{-1}(L) \quad \text { and } \quad q_{1}^{-1}(K) \cap s^{-1}(L)
$$

are compact.

(2) There exists $d \geq 0$ such that

$$
\left|q^{-1}(K) \cap s^{-1}(v)\right| \leq d \text { for all } v \in E^{0} .
$$

Proof. (1) follows immediately from Lemma 3.8, For (2), note first of all that the conclusion is unaffected by replacing $E$ with an isomorphic topological graph, so by Theorem 3.4 we may assume that $E$ is constructed from a topological graph $F$, a principal $G$-bundle $q: P \rightarrow F^{0}$, and an isomorphism $\theta: s^{*}(P) \rightarrow r^{*}(P)$. Thus $E^{1}=s^{*}(P)$, with quotient maps $\pi_{1}: s^{*}(P) \rightarrow F^{1}$ on edges and $q: P \rightarrow F^{0}$ on vertices, and source map $\pi_{2}: s^{*}(P) \rightarrow P$. Note that for $v \in P$, if we put $u=q(v) \in F^{0}$ then we have

$$
\pi_{1}^{-1}(K) \cap \tilde{s}^{-1}(v)=\left\{(e, v) \in s^{*}(P): e \in K\right\}=\left(K \cap s^{-1}(u)\right) \times\{v\} .
$$

Thus

$$
\left|\pi_{1}^{-1}(K) \cap \tilde{s}^{-1}(v)\right|=\left|K \cap s^{-1}(u)\right| .
$$


Since $s: F^{1} \rightarrow F^{0}$ is a local homeomorphism and $K$ is compact, the cardinalities of the intersections $K \cap s^{-1}(u)$ for $u \in F^{0}$ are bounded above by some fixed real number.

Remark 3.10. If $G$ is discrete and $G$ acts freely and properly on $E$, then the morphism $q: E \rightarrow E / G$ is a graph covering, since $G$ acts properly discontinuously (see 4.69 in [RW98).

Remark 3.11. If $E$ and $G$ are discrete and $G$ acts freely on $E$, then by Theorem 2.2.2 in GT01, there is a cocycle $c:(E / G)^{1} \rightarrow G$ such that $(E / G) \times_{c} G \cong E$ in an equivariant way. This result can be obtained from Corollary 3.7, since any principal $G$-bundle over $E^{0}$ is trivial. In general, not every free and proper action on a topological graph is associated to a skew product.

Recall that by [Kat06, Proposition 8.9] a topological graph $E$ is minimal iff every orbit space $\operatorname{Orb}(v, e)$ (see Kat06. Definition 4.9]) is dense in $E^{0}$, where $v \in E^{0}$ and $e$ is a negative orbit of $v$. If $E^{0}=E_{\mathrm{rg}}^{0}$, a negative orbit of $v$ is an infinite path $e=e_{1} e_{2} \cdots \in E^{\infty}$ ending at $v$ and

$$
\operatorname{Orb}(v, e)=\left\{r\left(e^{\prime}\right) \mid e^{\prime} \in E^{m}, s\left(e^{\prime}\right)=s\left(e_{n}\right) \text { for some } n\right\} .
$$

Theorem 3.12. Let $E$ be a minimal topological graph and let $q: E \rightarrow F$ be a graph covering. Suppose that $E^{0}$ is not discrete and $E^{0}=E_{r g}^{0}$. Then $F$ is also minimal, $F^{0}$ is not discrete and $F^{0}=F_{r g}^{0}$. In particular, both $C^{*}(E)$ and $C^{*}(F)$ are simple.

Proof. Indeed, the unique path lifting property ensures that every orbit space in $F$ is dense. The second part follows from Corollary 8.13 in [Kat06.

Corollary 3.13. Let $E$ be a minimal topological graph and suppose that $E^{0}$ is not discrete and $E^{0}=E_{r g}^{0}$. If the discrete group $G$ acts freely and properly on $E$, then $C^{*}(E / G)$ is simple.

Example 3.14. Now let $E=E(G, S)$ be as in Example 2.5 where $G=\mathbb{R}$ and $S=\{1, \theta\}$, with $\theta$ is irrational. Then $E^{0}=\mathbb{R}, E^{1}=\{1, \theta\} \times \mathbb{R}$. Translation by $\mathbb{Z}$ gives a free and proper action on both $E^{0}$ and $E^{1}$ which intertwines the structure maps $r, s$. Hence, translation induces a free and proper action of $\mathbb{Z}$ on $E$. Since $E$ is a minimal topological graph, $E^{0}$ is not discrete and $E^{0}=E_{\mathrm{rg}}^{0}$, we have by the above corollary that $C^{*}(E / \mathbb{Z})$ is simple.

\section{The Fundamental group of a topological GRAPH}

In this section, we define the fundamental group of a topological graph $E=$ $\left(E^{0}, E^{1}, s, r\right)$ and the notion of a universal covering of $E$, using a single topological space $R(E)$ called the geometric realization. We need to assume that the geometric realization has a universal covering. In this section we need not assume that the source map is a local homeomorphism, even though in all of our examples this is the case.

For each $e \in E^{1}$ we formally denote the reversed edge by $\bar{e}$, where $s(\bar{e})=r(e)$ and $r(\bar{e})=s(e)$. The set of reversed edges is denoted $\bar{E}^{1}$. A walk in $E$ is a sequence $w=e_{1} \cdots e_{n}$ where $e_{i} \in E^{1} \cup \bar{E}^{1}$ such that $s\left(e_{i}\right)=r\left(e_{i+1}\right)$ for $i=1, \ldots, n-1$. We define $s(w):=s\left(e_{n}\right)$ and $r(w):=r\left(e_{1}\right)$. A vertex will be considered as a trivial walk. A walk $w$ is reduced if it does not contain the subword $e \bar{e}$ for any $e \in E^{1} \cup \bar{E}^{1}$. 
Denote by $E^{r w}$ the space of reduced walks, with product topology. Note that $E^{0}$ is viewed as a subset of $E^{r w}$. For $w=e_{1} \cdots e_{n} \in E^{r w}$, the reverse walk $\bar{w}$ is $\bar{e}_{n} \cdots \bar{e}_{1}$.

The following definition is modeled on [BH99, Definition III.G.3.1].

Definition 4.1. An $E$-path $c=\left(w_{0}, c_{1}, w_{1}, \ldots, c_{k}, w_{k}\right)$ over a partition $0=t_{0} \leq$ $\ldots \leq t_{k}=1$ of the interval $[0,1]$ consists of continuous maps $c_{i}:\left[t_{i-1}, t_{i}\right] \rightarrow E^{0}$ and reduced walks $w_{i} \in E^{r w}$ such that $r\left(w_{i}\right)=c_{i+1}\left(t_{i}\right)$ for $i=0,1, \ldots, k-1$ and $s\left(w_{i}\right)=c_{i}\left(t_{i}\right)$ for $i=1,2, \ldots, k$. The initial point of $c$ is $x=s\left(w_{0}\right)$; its terminal point is $y=r\left(w_{k}\right)$. We say that $c$ joins $x$ to $y$. If $w_{0}$ and $w_{k}$ are trivial walks (vertices), they can be dropped in the notation for $c$. In particular, a map $c:[0,1] \rightarrow E^{0}$ can be considered as an $E$-path.

Definition 4.2. A topological graph $E$ is said to be connected if given any two vertices $x, y \in E^{0}$, there is an $E$-path $c$ joining $x$ and $y$.

Definition 4.3. The geometric realization of a topological graph $E$ is the topological space $R(E)$ obtained from the disjoint union $E^{1} \times[0,1] \sqcup E^{0}$ by identifying $(e, 0)$ with $s(e)$ and $(e, 1)$ with $r(e)$ (a kind of double mapping torus). We will identify $E^{0}$ with a subspace of $R(E)$ in the obvious way. Also, we embed $E^{1}$ in $R(E)$ by $e \in E^{1} \mapsto\left(e, \frac{1}{2}\right)$.

Remark 4.4. Notice that if $E$ is connected, then $R(E)$ is path connected. Let $\varphi: E \rightarrow F$ be a graph morphism. Then $\varphi$ yields a natural map

$$
E^{1} \times[0,1] \sqcup E^{0} \rightarrow F^{1} \times[0,1] \sqcup F^{0}
$$

which then induces a map $R(\varphi): R(E) \rightarrow R(F)$. Moreover, for $i=0,1$ the following diagram commutes:

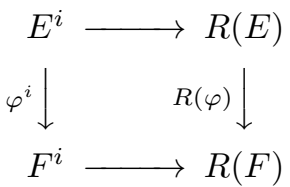

where the horizontal maps are the canonical embeddings given in Definition 4.3 . Observe that $R(\varphi)$ is continuous; moreover, $R(\varphi)$ is a covering if $\varphi$ is.

Remark 4.5. If the group $G$ acts on the topological graph $E$, then $G$ acts on $R(E)$ by $g \cdot(e, t)=\left(\lambda_{g}^{1}(e), t\right)$ for $e \in E^{1}, t \in[0,1]$, and by $g \cdot v=\lambda_{g}^{0}(v)$ for $v \in E^{0}$. Since $\lambda_{g}^{i}$ commute with $s$ and $r$ for $i=0,1$, the action is well defined.

Definition 4.6. Let $E=\left(E^{0}, E^{1}, s, r\right)$ be a a connected topological graph. We define the fundamental group of $E$ by $\pi_{1}(E):=\pi_{1}(R(E))$. We say that $E$ is simply connected if $\pi_{1}(E)$ is trivial (i.e. $R(E)$ is simply connected). We say that a graph morphism $p: \tilde{E} \rightarrow E$ is a universal covering if $p$ is a covering and $\tilde{E}$ is connected and simply connected (or briefly that $\tilde{E}$ is a universal cover).

Remark 4.7. The fundamental group $\pi_{1}(E)$ acts freely on $\tilde{E}$, and the orbit space is isomorphic to $E$. Any subgroup $H$ of $\pi_{1}(E)$ will determine an intermediate covering of $E$, by taking the graph $\tilde{E} / H$. Recall that the fundamental group of a finite graph $E$ is the free group with $\left|E^{1}\right|-\left|E^{0}\right|+1$ generators, see [KP99, Lemma 4.10].

Proposition 4.8. Let $E$ be a connected topological graph and suppose that $R=$ $R(E)$ is locally path-connected and semi-locally simply connected. Then $R$ has a 
universal covering space $\tilde{R}$. Moreover, $\tilde{R} \cong R(\tilde{E})$ where $\tilde{E}$ is a universal cover of E.

Proof. By [Mas91, Theorem 10.2] $R$ has a universal covering space $\tilde{R}$. Let $\pi: \tilde{R} \rightarrow$ $R$ be the canonical map, let $\tilde{E}^{0}=\pi^{-1}\left(E^{0}\right)$, and let $\tilde{E}^{1}=\pi^{-1}\left(E^{1} \times\{1 / 2\}\right)$. In order to define the range and the source maps, we use the unique path lifting property of the map $\pi$. Let $\tilde{e} \in \tilde{E}^{1}$, then $\pi(\tilde{e})=(e, 1 / 2)$ for some $e \in E^{1}$. Join $(e, 1 / 2)$ with the image of $(e, 0)$ in $R$ by the path $t \mapsto\left(e, \frac{1}{2}(1-t)\right), t \in[0,1]$. Lift this path to a path in $\tilde{R}$, and define $s(\tilde{e})$ to be the endpoint of the lifted path, which belongs to $\tilde{E}^{0}$. The range map is obtained similarly by joining $(e, 1 / 2)$ with the image of $(e, 1)$.

Corollary 4.9. Consider a connected topological graph $E$. If both $r, s: E^{1} \rightarrow E^{0}$ are finite-to-one covering maps and $E^{0}$ is locally contractible (each point has a local base of contractible neighborhoods), then $R(E)$ is also locally contractible. In particular, E has a universal cover.

Proof. It suffices to show that $R(E)$ is locally contractible since the hypotheses of the above proposition will be satisfied. A point $(e, t)$ in the image of $E^{1} \times(0,1)$ has a local base of contractible neighborhoods of the form $V \times W$, where $V$ is a contractible neighborhood of $e$ in $E^{1}$ and $W$ is a contractible neighborhood of $t$ in $(0,1)$. For the image of $v \in E^{0}$ in $R(E)$, take $V \subset E^{0}$ a contractible neighborhood of $v$ which is evenly covered by the maps $s$ and $r$. Then we claim that the image $N$ of $s^{-1}(V) \times[0, \varepsilon) \cup r^{-1}(V) \times(1-\varepsilon, 1]$ in $R(E)$ is a contractible neighborhood of the image of $v$ in $R(E)$ for any $0<\varepsilon<1 / 2$. Indeed, $s^{-1}(V)$ is a disjoint union $V_{1} \cup \ldots \cup V_{p}$, and $r^{-1}(V)$ is a disjoint union $U_{1} \cup \ldots \cup U_{q}$ of homeomorphic copies of $V$. Let $S$ be the star with $p+q$ branches obtained by gluing $p+q$ copies of $[0, \varepsilon)$ at 0 . Then $N$ is homeomorphic to $S \times V$, which is contractible.

It would be nice to have a weaker condition on $E$ that would ensure the existence of the universal cover.

Remark 4.10. In order to obtain other coverings of a graph $E$, we may consider a subgroup $H$ of the fundamental group $\pi_{1}(E)$ which will act on $\tilde{R}$, and take the corresponding topological graph of the quotient space $\tilde{R} / H$, constructed as in the proof of Proposition 4.8 .

Remark 4.11. The fundamental group of $\left(E^{0}, E^{1}, s, r\right)$ is isomorphic to the fundamental group of the opposite graph $\left(E^{0}, E^{1}, r, s\right)$, obtained by interchanging the maps $s$ and $r$. The natural embeddings described in 4.3 induce maps $\pi_{1}\left(E^{0}, v\right) \rightarrow$ $\pi_{1}(E)$ and $\pi_{1}\left(E^{1}, e\right) \rightarrow \pi_{1}(E)$ for fixed $v \in E^{0}$ and $e \in E^{1}$.

Example 4.12. Consider the topological graph $E$ with $E^{0}=E^{1}=\mathbb{T}$ and source and range maps $s(z)=z, r(z)=e^{2 \pi i \theta} z$ for $\theta$ irrational. The geometric realization is homeomorphic to the 2-torus $\mathbb{T}^{2}$, hence the fundamental group $\pi_{1}(E)$ is isomorphic to $\mathbb{Z}^{2}$. The universal covering graph is $\tilde{E}=\left(\tilde{E}^{0}, \tilde{E}^{1}, s, r\right)$, where $\tilde{E}^{0}=\tilde{E}^{1}=\mathbb{R} \times \mathbb{Z}$, with $s(y, k)=(y, k), r(y, k)=(y+\theta, k+1)$. The action of $\mathbb{Z}^{2}$ on $\tilde{E}$ is given by $(j, m) \cdot(y, k)=(j+y+m \theta, k+m)$, and $\tilde{E} / \mathbb{Z}^{2} \cong E$. Any other connected covering of $E$ is of the form $\tilde{E} / H$, where $H$ is a subgroup of $\mathbb{Z}^{2}$.

More generally, let $X$ be a compact space which admits a universal covering space $\tilde{X}$, and let $h: X \rightarrow X$ be a homeomorphism. The geometric realization of the corresponding topological graph $E$ with $E^{0}=E^{1}=X, s=i d$ and $r=h$ is 
homeomorphic to the mapping torus of $h$, obtained from $X \times[0,1]$ by identifying $(x, 1)$ with $(h(x), 0)$. The universal covering graph $\tilde{E}$ has $\tilde{E}^{0}=\tilde{E}^{1}=\tilde{X} \times \mathbb{Z}$. The source and range maps are given by $s(y, k)=(y, k), r(y, k)=(\tilde{h}(y), k+1)$, where $\tilde{h}: \tilde{X} \rightarrow \tilde{X}$ is a lifting of $h, y \in \tilde{X}$ and $k \in \mathbb{Z}$. The map $h$ induces an automorphism $h_{*}$ of $\pi_{1}(X)$, and the fundamental group of $E$ is isomorphic to the semi-direct product $\pi_{1}(X) \rtimes \mathbb{Z}$ defined using $h_{*}$. The action of $\pi_{1}(X) \rtimes \mathbb{Z}$ on $\tilde{X} \times \mathbb{Z}$ is given by $(g, m) \cdot(y, k)=\left(g \cdot \tilde{h}^{m}(y), k+m\right)$.

Example 4.13. Let again $E^{0}=E^{1}=\mathbb{T}$ with source and range maps $s(z)=$ $z^{n}, r(z)=z^{m}$ for $n, m$ positive integers. The geometric realization $R(E)$ is obtained from the cylinder $E^{1} \times[0,1]$, where the two boundary circles are identified using the source and range maps. Alternatively, we may start with a rectangle, such that the left and right edges are labeled by $a$. The top edge $E^{1} \times\{0\}$ is divided into $n$ segments called $b$, and the bottom edge $E^{1} \times\{1\}$ is divided into $m$ segments also called $b$. By making the identifications, we get the geometric realization $R(E)$. Note that here the arrows are for identification purposes.

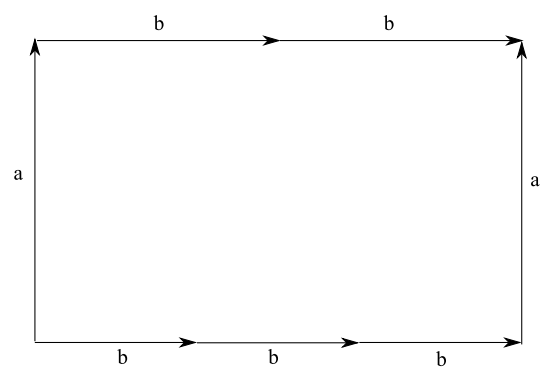

Figure 1 . The case $n=2, m=3$.

The segments $a, b$ become generators in the fundamental group $\pi_{1}(R(E))$, which is isomorphic to the Baumslag-Solitar group

$$
B(n, m)=\left\langle a, b \mid a b^{n} a^{-1}=b^{m}\right\rangle .
$$

For $n=1$ or $m=1$, this group is a semi-direct product and it is amenable. For $n \neq 1, m \neq 1$ and $n, m$ relatively prime, it is not amenable (see Example E.11 in BO08).

The universal covering space of $R(E)$ is the Cayley complex $\tilde{R}$ of $B(n, m)$, obtained from the Cayley graph by filling out the rectangles (see, for example page 122 in [LS77]). It is the cartesian product $T \times \mathbb{R}$, where $T$ is the Bass-Serre tree of $B(n, m)$, viewed as an HNN-extension. Recall that, given a group $G$, a subgroup $H \subset G$, and a monomorphism $\tau: H \rightarrow G$, then the HNN extension $G *_{H} \tau$ is generated by $G$ and a letter $a$ such that $a h a^{-1}=\tau(h)$ for $h \in H$ (see for example [Bau93]). In our case, $G=\langle b\rangle \cong \mathbb{Z}, H=\left\langle b^{n}\right\rangle \cong n \mathbb{Z}$, and $\tau\left(b^{n}\right)=b^{m}$.

The free action of the group $B(n, m)$ on $\tilde{R}$ is the extension to the 2-cells of the usual left action on the Cayley graph. A piece of the Cayley complex of $B(2,3)$ together with the action of the generators is illustrated in Figure 2.

The 1-skeleton is the directed Cayley graph of $B(2,3)$, where the generators $a, b$ multiply on the right. The group action is given by left multiplication. In the 


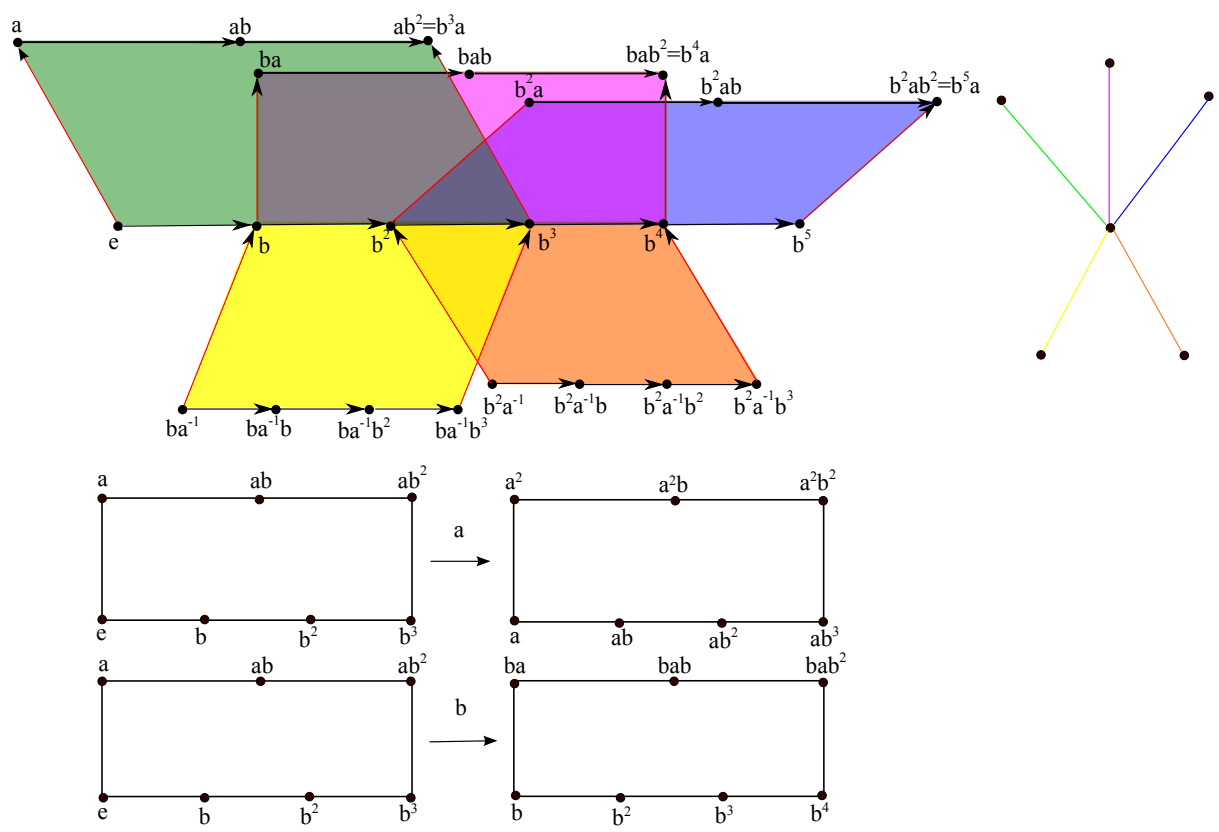

Figure 2. Cayley complex for $B(2,3)$.

corresponding tree $T$, each vertex has 5 edges. The vertex set $T^{0}$ is identified with the left cosets $g\langle b\rangle \in B(2,3) /\langle b\rangle$, and the edge set $T^{1}$ with the left cosets $g\left\langle b^{2}\right\rangle \in$ $B(2,3) /\left\langle b^{2}\right\rangle$. The source and range maps are given by $s\left(g\left\langle b^{2}\right\rangle\right)=g\langle b\rangle, r\left(g\left\langle b^{2}\right\rangle\right)=$ $g a^{-1}\langle b\rangle$ for $g \in B(2,3)$.

Using Proposition 4.8 we can describe the universal covering graph $\tilde{E}$ of $E$. We have $\tilde{E}^{0} \cong T^{0} \times \mathbb{R}, E^{1} \cong T^{1} \times \mathbb{R}$ with $\tilde{s}(t, y)=(s(t), n y), \tilde{r}(t, y)=(r(t), m y)$ for $t \in T^{1}$ and $y \in \mathbb{R}$. The group $B(n, m)$ acts freely and properly on $\tilde{E}$, and the quotient graph $\tilde{E} / B(n, m)$ is isomorphic to $E$. In particular, note that the topological graph $\tilde{E}$ is not a skew product. In the next section, we show that $C^{*}(\tilde{E}) \rtimes_{r} B(n, m)$ is strongly Morita equivalent to $C^{*}(E)$.

\section{Group aCtions on $C^{*}$-CORRESpondences}

Definition 5.1. Let $G$ be a locally compact group, and let $\mathcal{H}$ be a $C^{*}$-correspondence over $A$. We say that $G$ acts on $\mathcal{H}$ if there is a map

$$
G \times \mathcal{H} \rightarrow \mathcal{H}, \quad(g, \xi) \mapsto g \cdot \xi
$$

such that $g \mapsto g \cdot \xi$ is continuous and $\xi \mapsto g \cdot \xi$ is linear, and a continuous action of $G$ on $A$ by *-automorphisms such that

$$
\langle g \cdot \xi, g \cdot \eta\rangle=g \cdot\langle\xi, \eta\rangle, g \cdot(\xi a)=(g \cdot \xi)(g \cdot a), g \cdot(\varphi(a) \xi)=\varphi(g \cdot a)(g \cdot \xi),
$$

where $\varphi: A \rightarrow \mathcal{L}(\mathcal{H})$ defines the left multiplication.

Remark 5.2. There is an action of $G$ on $\mathcal{L}(\mathcal{H})$ given by

$$
(g \cdot T)(\xi)=g \cdot\left(T\left(g^{-1} \cdot \xi\right)\right),
$$


such that $g \mapsto g \cdot T$ is continuous with respect to the strict topology on $\mathcal{L}(\mathcal{H})$. We have $g \cdot\left(\theta_{\xi, \eta}\right)=\theta_{g \cdot \xi, g \cdot \eta}$, which gives an action on $\mathcal{K}(\mathcal{H})$. Indeed,

$$
g \cdot\left(\theta_{\xi, \eta}\right)(\zeta)=g \cdot\left(\xi\left\langle\eta, g^{-1} \cdot \zeta\right\rangle\right)=(g \cdot \xi)\langle g \cdot \eta, \zeta\rangle=\left(\theta_{g \cdot \xi, g \cdot \eta}\right)(\zeta) .
$$

An action of $G$ on the $C^{*}$-correspondence $\mathcal{H}$ defines an action on the Toeplitz algebra $\mathcal{T}_{\mathcal{H}}$ by $g \cdot T_{\xi}=T_{g \cdot \xi}$, and on the Cuntz-Pimsner algebra $\mathcal{O}_{\mathcal{H}}$, since all defining relations are equivariant.

The full crossed product $\mathcal{H} \rtimes G$ can be defined by $\mathcal{H} \rtimes G=\mathcal{H} \otimes_{\varphi}(A \rtimes G)$, where $\varphi: A \rightarrow \mathcal{L}(A \rtimes G)$ is the embedding of $A$ in the multiplier algebra of the crossed product $A \rtimes G$, regarded as a Hilbert module over itself.

Note that $G$ acts on $\mathcal{H} \rtimes G$ by $g \cdot(\xi \otimes f)=g \cdot \xi \otimes\left(u_{g} f u_{g}^{-1}\right)$, where $\xi \in \mathcal{H}, f \in A \rtimes G$, and $g \cdot a=u_{g} a u_{g}^{-1}$. Here $u: G \rightarrow U M(A \rtimes G)$ is the canonical map into the unitary multipliers. The crossed product $\mathcal{H} \rtimes G$ becomes a $C^{*}$-correspondence over $A \rtimes G$ in a natural way.

Similarly, the reduced crossed product $\mathcal{H} \rtimes_{r} G$ can be defined as a $C^{*}$-correspondence over $A \rtimes_{r} G$; moreover, there is a natural action of $G$ on $\mathcal{H} \rtimes_{r} G$ defined as above.

Remark 5.3. This crossed product $\mathcal{H} \rtimes G$ is isomorphic to the one defined using a completion of $C_{c}(G, \mathcal{H})$, as in Kas88 and [HN08. The isomorphism is induced by the function

$$
\mathcal{H} \otimes C_{c}(G, A) \rightarrow C_{c}(G, \mathcal{H})
$$

which sends $\xi \otimes f$ to the map $g \mapsto \xi f(g)$. It is proved in [HN08, Theorem 2.10] that if $G$ is amenable, then

$$
\mathcal{O}_{\mathcal{H} \rtimes G} \cong \mathcal{O}_{\mathcal{H}} \rtimes G .
$$

Note that we also consider actions and crossed products by non-amenable groups.

Proposition 5.4. If $G$ acts on the topological graph $E=\left(E^{0}, E^{1}, s, r\right)$, then $G$ acts on the $C^{*}$-correspondence $\mathcal{H}=\mathcal{H}(E)$ and hence on $C^{*}(E)$.

Proof. Define $g \cdot \xi(e)=\xi\left(g^{-1} e\right)$ for $\xi \in C_{c}\left(E^{1}\right)$, and $g \cdot f(v)=f\left(g^{-1} v\right)$ for $f \in$ $C_{0}\left(E^{0}\right)$. Then this action is compatible with the bimodule structure since $s$ and $r$ are equivariant.

Definition 5.5. Recall from Rie90 that the action $\alpha$ of a locally compact group $G$ on a $C^{*}$-algebra $A$ is proper if there is a dense $\alpha$-invariant *-subalgebra $A_{0}$ of $A$ such that for every $a, b \in A_{0}$ the functions

$$
x \mapsto a \alpha_{x}(b) \text { and } x \mapsto \Delta(x)^{-1 / 2} a \alpha_{x}(b)
$$

are integrable and there exists a (right) inner product $\langle\cdot, \cdot\rangle_{r}$ with values in the subalgebra of $M(A)$, which Rieffel denotes by $M\left(A_{0}\right)$, comprising those elements that multiply $A_{0}$ into itself, such that

$$
c\langle a, b\rangle_{r}=\int_{G} c \alpha_{x}\left(a^{*} b\right) d x \text { for all } c \in A_{0} .
$$

For such an action,

$$
A^{\alpha}:=\overline{\operatorname{span}}\left\{\langle a, b\rangle_{r}: a, b \in A_{0}\right\} \subset M(A)
$$

is called the generalized fixed-point algebra. Define a (left) inner product on $A_{0}$ with values in $A \rtimes_{\alpha, r} G$ by

$$
\ell\langle a, b\rangle(x)=\Delta(x)^{-1 / 2} a \alpha_{x}\left(b^{*}\right) .
$$


The set

$$
I:=\overline{\operatorname{span}}\left\{\ell\langle a, b\rangle: a, b \in A_{0}\right\}
$$

is an ideal in $A \rtimes_{\alpha, r} G$, and the closure $\mathcal{Z}$ of $A_{0}$ in the norm $\|a\|^{2}:=\left\|\langle a, a\rangle_{r}\right\|$ is an $I-A^{\alpha}$ imprimitivity bimodule. The action is called saturated if $I=A \rtimes_{\alpha, r} G$.

We review now the averaging process of KQR08, Section 2], which was built upon work in OP78, OP80, Qui92, QR95. Let a locally compact group $G$ act freely and properly on a locally compact Hausdorff space $T$, and let $\gamma$ be the associated action of $G$ on $C_{0}(T)$ :

$$
\left(\gamma_{g}(f)\right)(t)=f\left(g^{-1} \cdot t\right) \quad \text { for } \quad g \in G, f \in C_{0}(T), t \in T .
$$

Also let $\alpha$ be an action of $G$ on a $C^{*}$-algebra $A$, and suppose that we have an equivariant nondegenerat ${ }^{1}$ homomorphism $\varphi: C_{0}(T) \rightarrow M(A)$. For $g \in G$ let $\overline{\alpha_{g}}$ denote the canonical extension of $\alpha_{g}$ to $M(A)$, and write $M(A)^{\alpha}=\{a \in M(A)$ : $\overline{\alpha_{g}}(a)=a$ for all $\left.g \in G\right\}$ (not to be confused with Rieffel's generalized fixed-point algebras discussed above). It was shown in $\mathrm{KQR08}$ that

$$
A_{0}:=\operatorname{span} \varphi\left(C_{c}(T)\right) A \varphi\left(C_{c}(T)\right)
$$

is a dense $*$-subalgebra of $A$, and that there is a linear map $\Phi: A_{0} \rightarrow M(A)^{\alpha}$ such that

$$
\omega(\Phi(a))=\int_{G} \omega\left(\alpha_{g}(a)\right) d g \text { for all } a \in A_{0}, \omega \in A^{*} .
$$

We write $\Phi^{\alpha}$ when confusion is possible. KQR08 also shows that $\Phi\left(A_{0}\right)$ is a *-subalgebra of $M(A)$, and so its norm closure, denoted by $\operatorname{Fix}(A, \alpha, \varphi)$, is a $C^{*}$ subalgebra.

[Rie04, Theorem 5.7] implies that the action $\alpha$ is proper and saturated with respect to $A_{0}$, and so by Rie90 the reduced crossed product $A \times_{\alpha, r} G$ is Morita equivalent to a generalized fixed-point algebra $A^{\alpha}$. It was shown in KQR08, Proposition 3.1] that $\operatorname{Fix}(A, \alpha, \varphi)$ coincides with the algebra $A^{\alpha}$ of [Rie90].

We record a few properties of this averaging process, which can be found in KQR08, Section 2]:

- If $a \in A_{0}$ and $b \in M(A)^{\alpha}$ then $a b \in A_{0}$ and $\Phi(a b)=\Phi(a) b$.

- If $f \in C_{c}(T)$ and $a \in A_{0}$ then the function $g \mapsto \varphi(f) \alpha_{g}(a)$ belongs to $C_{c}(G, A)$ and

$$
\int_{G} \varphi(f) \alpha_{g}(a) d g=\varphi(f) \Phi(a) .
$$

- If $(B, \beta)$ is another action, and $\psi: C_{0}(T) \rightarrow M(B)$ and $\sigma: A \rightarrow M(B)$ are nondegenerate and $G$-equivariant, and if the canonical extension $\bar{\sigma}$ : $M(A) \rightarrow M(B)$ satisfies $\bar{\sigma} \circ \phi=\psi$, then $\bar{\sigma}$ restricts to a nondegenerate homomorphism

$$
\bar{\sigma} \mid: \operatorname{Fix}(A, \alpha, \varphi) \rightarrow M(\operatorname{Fix}(B, \beta, \psi)) .
$$

Moreover,

$$
\Phi^{\beta} \circ \sigma=\bar{\sigma} \circ \Phi^{\alpha} .
$$

- For fixed $f, h \in C_{c}(T)$ the map

$$
a \mapsto \Phi(\varphi(f) a \varphi(h)): A_{0} \rightarrow M(A)
$$

is norm continuous.

\footnotetext{
${ }^{1}$ recall that this means that $\varphi\left(C_{0}(T)\right) A=A$
} 
For the proof of Theorem 5.6 below, we will need to make extensive use of the multiplier bimodules of EKQR06; the relevant facts are collected in Appendix A.

Theorem 5.6. If a locally compact group $G$ acts freely and properly on a topological graph $E$, then $C^{*}(E) \rtimes_{r} G$ and $C^{*}(E / G)$ are strongly Morita equivalent.

Proof. Since $G$ acts freely and properly on $E^{0}$ and there is a nondegenerate equivariant homomorphism $C_{0}\left(E^{0}\right) \rightarrow M\left(C^{*}(E)\right)$, it follows from Rie04 and Lemma 4.1 in HRW05 that the corresponding action $\alpha$ of $G$ on $C^{*}(E)$ is proper and saturated with respect to the $*$-subalgebra $A_{0}=C_{c}\left(E^{0}\right) C^{*}(E) C_{c}\left(E^{0}\right)$ of $C^{*}(E)$. Therefore the reduced crossed product $C^{*}(E) \rtimes_{r} G$ is strongly Morita equivalent to the generalized fixed point algebra $C^{*}(E)^{\alpha}$. Thus it suffices to show $C^{*}(E)^{\alpha} \cong C^{*}(E / G)$, and for this we will construct an injective homomorphism from $C^{*}(E / G)$ to $M\left(C^{*}(E)\right)$ whose image is $C^{*}(E)^{\alpha}$.

As before, denote the quotient topological graph by $F=E / G$, so that $F^{0}=$ $E^{0} / G$ and $F^{1}=E^{1} / G$. It will also be convenient to denote both quotient maps $E^{0} \rightarrow F^{0}$ and $E^{1} \rightarrow F^{1}$ by $q$ (and no confusion will occur since the particular $q$ that is intended will be clear from the context), and to use the following abbreviations:

$$
\begin{aligned}
& \text { - } A=C_{0}\left(E^{0}\right) \\
& \text { - } B=C_{0}\left(F^{0}\right) \\
& \text { - } X=\mathcal{H}(E) \\
& \text { - } Y=\mathcal{H}(F)
\end{aligned}
$$

Of course, our homomorphism $C^{*}(F) \rightarrow M\left(C^{*}(E)\right)$ will be of the form $\tau \times \pi$ for a Cuntz-Pimsner covariant Toeplitz representation $(\tau, \pi)$ of $Y$ in $M\left(C^{*}(E)\right)$.

We will construct $(\tau, \pi): Y \rightarrow M\left(C^{*}(E)\right)$ by, roughly speaking, first mapping the $B$-correspondence $Y$ into the multiplier bimodule $M(X)$, then composing with the canonical Toeplitz representation $\left(k_{X}, k_{A}\right)$ of the $A$-correspondence $X$ in $C^{*}(E)$. Actually, this needs a little tweaking; our strategy is more accurately indicated by the diagram

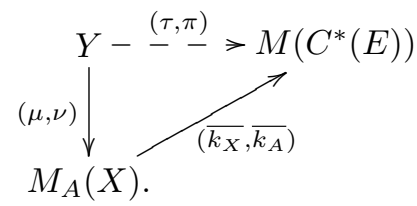

Note the appearance in the above diagram of the " $A$-multiplier" bimodule $M_{A}(X)$ (see Definition A.8 and Remark A.10; this is necessary because the canonical Toeplitz representation $\left(k_{X}, k_{A}\right)$ can be degenerate - see Example A.1.

Our proof is rather long, and to improve readability we break it into the following steps:

(1) construct a correspondence homomorphism $(\mu, \nu)$ as in Diagram 3

(2) define $(\tau, \pi)$ to make Diagram 3 commute, and show that it is a Toeplitz representation;

(3) show that the Toeplitz representation $(\tau, \pi)$ is Cuntz-Pimsner covariant;

(4) show that the associated homomorphism $\tau \times \pi: C^{*}(F) \rightarrow M\left(C^{*}(E)\right)$ is injective;

(5) show that the image of $\tau \times \pi$ is $C^{*}(E)^{\alpha}$.

We take each step in order: 
Step 1. Clearly, we should define $(\mu, \nu)$ by composing with the quotient map $q$. On $B$ this obviously does the right thing:

$$
\nu: B \rightarrow M(A), \quad \nu(f):=f \circ q .
$$

On $Y$, things are a little more delicate: for $\xi \in C_{c}\left(F^{1}\right)$ and $f \in C_{c}\left(E^{0}\right)$ define $\mu(\xi) \cdot f: E^{1} \rightarrow \mathbb{C}$ by

$$
(\mu(\xi) \cdot f)(e)=\xi(q(e)) f(s(e)) .
$$

Then $\mu(\xi) \cdot f \in C_{c}\left(E^{1}\right)$ by Lemma 3.9

We must show that we can extend $\mu$ to get a correspondence homomorphism $(\mu, \nu): Y \rightarrow M_{A}(X)$, and we will accomplish this by:

- extending to a correspondence homomorphism into $M(X)$, and then

- showing that it actually takes values in $M_{A}(X)$.

We first need to know that for $\xi \in C_{c}\left(F^{1}\right)$ the map

$$
f \mapsto \mu(\xi) \cdot f: C_{c}\left(E^{0}\right) \rightarrow C_{c}\left(E^{1}\right)
$$

extends to an adjointable map $A \rightarrow X$, i.e., an element of $M(X)$. For technical purposes, we need the following

Claim: If $\xi, \eta \in C_{c}\left(F^{1}\right)$ and $v \in E^{0}$, then

$$
\langle\xi, \eta\rangle(q(v))=\sum_{s(e)=v} \overline{\xi(q(e))} \eta(q(e)) .
$$

To see this, just observe that, for every $v^{\prime} \in F^{0}$, no matter which element $v \in$ $q^{-1}\left(v^{\prime}\right)$ we choose, the set of values

$$
\{\overline{\xi(q(e))} \eta(q(e)): s(e)=v\}
$$

coincides with the set of values

$$
\left\{\overline{\xi\left(e^{\prime}\right)} \eta\left(e^{\prime}\right): s\left(e^{\prime}\right)=v^{\prime}\right\},
$$

because $G$ acts freely and the source map $s: E^{1} \rightarrow E^{0}$ is $G$-equivariant, and we have proved the claim.

We use this to show that the linear map

$$
f \mapsto \mu(\xi) \cdot f: C_{c}\left(E^{0}\right) \rightarrow X
$$

is bounded, and hence extends uniquely to a bounded linear map

$$
\mu(\xi): A \rightarrow X .
$$

For $v \in E^{0}$ we have

$$
\begin{aligned}
\langle\mu(\xi) \cdot f, \mu(\xi) \cdot f\rangle(v) & =\sum_{s(e)=v}|\mu(\xi)(q(e))|^{2}|f(v)|^{2} \\
& =\langle\xi, \xi\rangle(q(e))|f(v)|^{2} \\
& \leq\|\xi\|^{2}\|f\|^{2}
\end{aligned}
$$

so

$$
\|\mu(\xi) \cdot f\| \leq\|\xi\|\|f\|,
$$

verifying the boundedness. 
We need to show that the linear map $\mu(\xi): A \rightarrow X$ is adjointable. For $\eta \in$ $C_{c}\left(E^{1}\right)$ define $\mu(\xi)^{*} \cdot \eta: E^{0} \rightarrow \mathbb{C}$ by

$$
\left(\mu(\xi)^{*} \cdot \eta\right)(v)=\sum_{s(e)=v} \overline{\xi(q(e))} \eta(e),
$$

where the sum is finite by Corollary 3.9 An argument similar to the proof of [Kat04, Lemma 1.5] shows that $\mu(\xi)^{*} \cdot \eta \in C_{c}\left(E^{0}\right)$. We have

$$
\begin{aligned}
\left|\left(\mu(\xi)^{*} \cdot \eta\right)(v)\right|^{2} & \leq \sum_{s(e)=v}|\xi(q(e))|^{2} \sum_{s(e)=v}|\eta(e)|^{2} \\
& =\langle\xi, \xi\rangle(q(v))\langle\eta, \eta\rangle(v) \\
& \leq\|\xi\|^{2}\|\eta\|^{2}
\end{aligned}
$$

so

$$
\left\|\mu(\xi)^{*} \cdot \eta\right\| \leq\|\xi\|\|\eta\|
$$

Thus the map

$$
\eta \mapsto \mu(\xi)^{*} \cdot \eta: C_{c}\left(E^{1}\right) \rightarrow C_{c}\left(E^{0}\right)
$$

extends uniquely to a bounded linear map

$$
\mu(\xi)^{*}: X \rightarrow A
$$

For $f \in C_{c}\left(E^{0}\right), \eta \in C_{c}\left(E^{1}\right)$, and $v \in E^{0}$ we have

$$
\begin{aligned}
\langle\mu(\xi) \cdot f, \eta\rangle(v) & =\sum_{s(e)=v} \overline{\xi(q(e)) f(v)} \eta(e) . \\
& =\overline{f(v)}\left(\mu(\xi)^{*} \cdot \eta\right)(v)
\end{aligned}
$$

so

$$
\langle\mu(\xi) \cdot f, \eta\rangle=\left\langle f, \mu(\xi)^{*} \cdot \eta\right\rangle
$$

and it follows by continuity that $\mu(\xi)^{*}: X \rightarrow A$ is an adjoint of $\mu(\xi)$. Therefore $\mu(\xi) \in M(X)$.

We now have a linear map $\mu: C_{c}\left(F^{1}\right) \rightarrow M(X)$, and then the estimate (4) shows $\mu$ is bounded, hence extends uniquely to a bounded linear map $\mu: Y \rightarrow M(X)$. We can now show that the pair $(\mu, \nu)$ is a Toeplitz representation. First we show that for $\xi, \eta \in Y$ we have

$$
\langle\mu(\xi), \mu(\eta)\rangle=\nu(\langle\xi, \eta\rangle),
$$

and by continuity the following computation is sufficient: for $\xi, \eta \in C_{c}\left(F^{1}\right)$ and $f \in C_{c}\left(E^{0}\right)$ and $v \in E^{0}$ we have

$$
\begin{aligned}
(\langle\mu(\xi), \mu(\eta)\rangle f)(v) & =\left(\mu(\xi)^{*} \cdot \mu(\eta) \cdot f\right)(v) \\
& =\sum_{s(e)=v} \overline{\xi(q(e))}(\mu(\eta) \cdot f)(e) \\
& =\sum_{s(e)=v} \overline{\xi(q(e))} \eta(q(e)) f(s(e)) \\
& =\langle\xi, \eta\rangle(q(v)) f(v) \\
& =(\nu(\langle\xi, \eta\rangle) f)(v) .
\end{aligned}
$$

We next show that for $f \in B$ and $\xi \in Y$ we have

$$
\mu(f \cdot \xi)=\nu(f) \cdot \mu(\xi),
$$


and again by continuity the following computation suffices: for $f \in C_{c}\left(F^{0}\right), \xi \in$ $C_{c}\left(F^{1}\right), h \in C_{c}\left(E^{0}\right)$, and $e \in E^{1}$ we have

$$
\begin{aligned}
(\mu(f \cdot \xi) \cdot h)(e) & =(f \cdot \xi)(q(e)) h(s(e)) \\
& =f(r(q(e))) \xi(q(e)) h(s(e)) \\
& =f(q(r(e)))(\mu(\xi) \cdot h)(e) \\
& =\nu(f)(r(e))(\mu(\xi) \cdot h)(e) \\
& =(\nu(f) \cdot(\mu(\xi) \cdot h))(e) \\
& =((\nu(f) \cdot \mu(\xi)) \cdot h)(e) .
\end{aligned}
$$

We thus have a homomorphism $(\mu, \nu)$ from the $B$-correspondence $Y$ to the $M(A)$ correspondence $M(X)$, and it remains to show that for $\xi \in Y$ we actually have $\mu(\xi) \in M_{A}(X)$, i.e., for $f \in A$ we have

$$
f \cdot \mu(\xi) \in X
$$

(because $\mu(\xi) \cdot f \in X$ automatically since $\mu(\xi)$ is a module multiplier). By continuity it suffices to show that if $\xi \in C_{c}\left(F^{1}\right)$ and $f \in C_{c}\left(E^{0}\right)$ then

$$
f \cdot \mu(\xi) \in C_{c}\left(E^{1}\right) .
$$

For $h \in C_{c}\left(E^{0}\right)$ and $e \in E^{1}$ we have

$$
\begin{aligned}
((f \cdot \mu(\xi)) \cdot h)(e) & =(f \cdot(\mu(\xi) \cdot h))(e) \\
& =f(r(e))(\mu(\xi) \cdot h)(e) \\
& =f(r(e)) \xi(q(e)) h(s(e)),
\end{aligned}
$$

so the multiplier $f \cdot \mu(\xi)$ of $X$ coincides with the element of $C_{c}\left(E^{1}\right)$ given by

$$
e \mapsto f(r(e)) \xi(q(e))
$$

(which has compact support by Corollary 3.9.

Step 2. By Corollary A.14 the Toeplitz representation

$$
\left(k_{X}, k_{A}\right): X \rightarrow C^{*}(E)
$$

extends to a Toeplitz representation

$$
\left(\overline{k_{X}}, \overline{k_{A}}\right): M_{A}(X) \rightarrow M_{A}\left(C^{*}(E)\right),
$$

because $k_{A}$ is nondegenerate.

Now define $(\tau, \pi): Y \rightarrow M_{A}\left(C^{*}(E)\right)$ to make diagram (3) commute, i.e.,

$$
\tau:=\overline{k_{X}} \circ \mu \text { and } \pi:=\overline{k_{A}} \circ \nu .
$$

Then $(\tau, \pi)$ is a Toeplitz representation, being the composition of the correspondence homomorphism $(\mu, \nu)$ and the Toeplitz representation $\left(\overline{k_{X}}, \overline{k_{A}}\right)$.

Step 3. Cuntz-Pimsner covariance of $(\tau, \pi)$ will follow from an analogous property of the correspondence homomorphism $(\mu, \nu)$. Our strategy is illustrated by the 
diagram

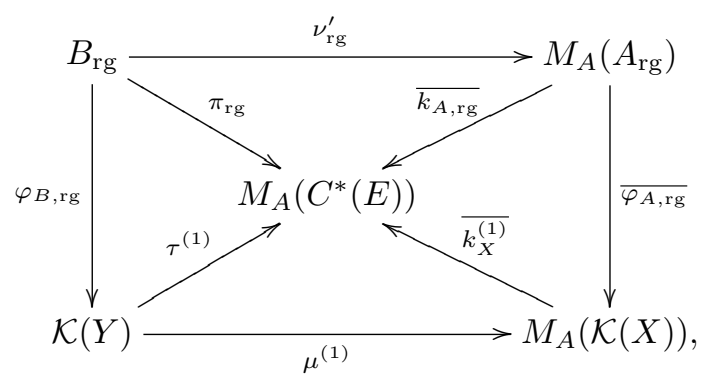

which requires explanation. We define $B_{\mathrm{rg}}=C_{0}\left(F_{\mathrm{rg}}^{0}\right)$, and we let $\varphi_{B \text {,rg }}$ denote the restriction to the ideal $B_{\mathrm{rg}}$ of the natural homomorphism $\varphi_{B}: B \rightarrow \mathcal{L}(Y)$ implementing the left module multiplication, and similarly for $A_{\mathrm{rg}}$ and $\varphi_{A, \mathrm{rg}}$. Also similarly for the restrictions $\pi_{\mathrm{rg}}$ of $\pi: B \rightarrow M_{A}\left(C^{*}(E)\right)$ and $k_{A, \mathrm{rg}}$ of $k_{A}: A \rightarrow$ $C^{*}(E)$. The bars in the right-hand triangle of (5) denote the unique $A$-strictly continuous extensions guaranteed by Lemma A.5 and Corollary A.7. For the bottom arrow, note that the correspondence homomorphism $\mu: Y \rightarrow M_{A}(X)$ naturally induces a homomorphism $\mu^{(1)}: \mathcal{K}(Y) \rightarrow \mathcal{K}\left(M_{A}(X)\right)$, and by Remark A.10 we have $\mathcal{K}\left(M_{A}(X)\right) \subset M_{A}(\mathcal{K}(X))$.

The part of (5) requiring the most explanation is $\nu_{\mathrm{rg}}^{\prime}$. We have defined a homomorphism $\nu: B \rightarrow M(A)$, and we denote the restriction to $B_{\mathrm{rg}}$ by

$$
\nu_{\mathrm{rg}}: B_{\mathrm{rg}} \rightarrow M(A)
$$

For the purposes of the rest of (5), what we need is to have a homomorphism into $M_{A}\left(A_{\mathrm{rg}}\right)$.

The problem is that $M_{A}\left(A_{\mathrm{rg}}\right)$ doesn't embed naturally in $M(A)$. However, we will show that $\nu_{\mathrm{rg}}$ actually maps into the $C^{*}$-subalgebra

$$
M_{A, A_{\mathrm{rg}}}(A):=\left\{f \in M(A): f A \subset A_{\mathrm{rg}}\right\}
$$

of $M(A)$. Since every $f \in M_{A, A_{\mathrm{rg}}}(A)$ vanishes on $E^{0} \backslash E_{\mathrm{rg}}^{0}$, the restriction map $\left.f \mapsto f\right|_{E_{\mathrm{rg}}^{\mathrm{o}}}$ gives an embedding of this subalgebra into $M_{A}\left(A_{\mathrm{rg}}\right)$, and we define $\nu_{\mathrm{rg}}^{\prime}$ so that the diagram

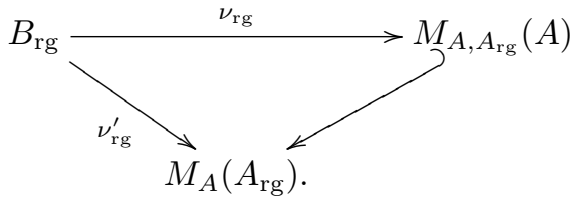

commutes.

The desired Cuntz-Pimsner covariance of $(\tau, \pi)$ follows from commutativity of the left triangle of (5) (see Remark 2.1), which we will deduce from that of the outer square and the other three triangles. 
The top triangle. The top triangle of (5) coincides with the lower left triangle of the diagram

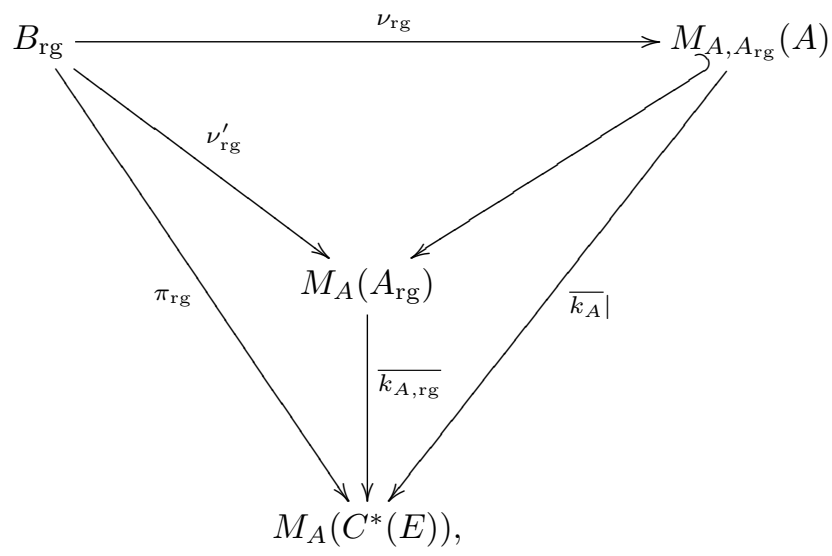

in which $\overline{k_{A}} \mid$ denotes the restriction of $\overline{k_{A}}: M(A) \rightarrow M_{A}\left(C^{*}(E)\right)$ to the subalgebra $M_{A, A_{\mathrm{rg}}}(A)$. Once we have verified that $\nu_{\mathrm{rg}}$ maps $B_{\mathrm{rg}}$ into $M_{A, A_{\mathrm{rg}}}(A)$, it will suffice to observe that all the other triangles in (6) commute: the top triangle of (6) commutes by definition of $\nu_{\mathrm{rg}}^{\prime}$, the outer triangle commutes by definition of $\pi_{\mathrm{rg}}$, and the lower-right triangle commutes by a routine check of the definitions.

We must show that if $f \in C_{0}\left(F_{\mathrm{rg}}^{0}\right)$ and $g \in C_{0}\left(E^{0}\right)$ then

$$
\nu(f) g=(f \circ q) g \in C_{0}\left(E_{\mathrm{rg}}^{0}\right) .
$$

We have $\nu(f) g \in C_{0}\left(E^{0}\right)$ since $\nu(f)$ is bounded and $g \in C_{0}\left(E^{0}\right)$. We must show that $\nu(f) g=0$ on $E^{0} \backslash E_{\mathrm{rg}}^{0}$, equivalently if $\nu(f) g$ is nonzero at an element $v \in E^{0}$, then we must have $v \in E_{\mathrm{rg}}^{0}$. So, we must show that $v$ is in the image of the range map $\tilde{r}: E^{1} \rightarrow E^{0}$, and that there is a neighborhood $U$ of $v$ such that $\tilde{r}^{-1}(U)$ is compact, i.e., $v \in E_{\text {fin }}^{0}$.

By Lemma 3.3 we may assume that $E^{1}=r^{*}\left(E^{0}\right)$, i.e., that the diagram

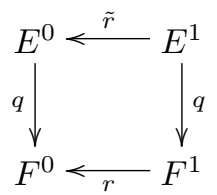

coincides with a pull-back

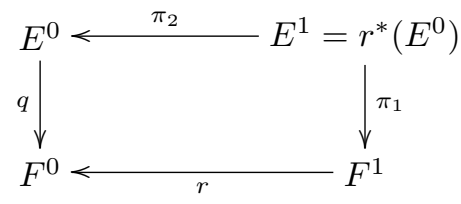

Since $\nu(f)(v) \neq 0$ and $f \in C_{0}\left(F_{\mathrm{rg}}^{0}\right)$, we have $q(v) \in F_{\mathrm{rg}}^{0}$. Thus we can choose $e \in F^{1}$ such that $r(e)=q(v)$. Then $(e, v) \in E^{1}=r^{*}\left(E^{0}\right)$ and $v=\pi_{2}(e, v)$, showing that $v$ is in the image of the range map on $E^{1}$.

To see that $v \in E_{\text {fin }}^{0}$, start with a compact neighborhood $U$ of $v$ in $E^{0}$, and put $V=q(U)$, which is a compact neighborhood of $q(v)$ in $F^{0}$. Shrink $U$ if necessary 
so that $W:=r^{-1}(V)$ is compact in $F^{1}$. We will show that $\pi_{2}^{-1}(U)$ is compact. Suppose $\pi_{2}(e, u) \in U$, i.e., $u \in U$ and $r(e)=q(u)$. Then $e \in W$. Thus

$$
(e, u) \in(W \times U) \cap r^{*}\left(E^{0}\right),
$$

which is compact. We have verified the inclusion $\nu_{\mathrm{rg}}\left(B_{\mathrm{rg}}\right) \subset M_{A, A_{\mathrm{rg}}}(A)$.

The right triangle. Cuntz-Pimsner covariance of the canonical homomorphism $\left(k_{X}, k_{A}\right)$ is expressed by the commutative diagram

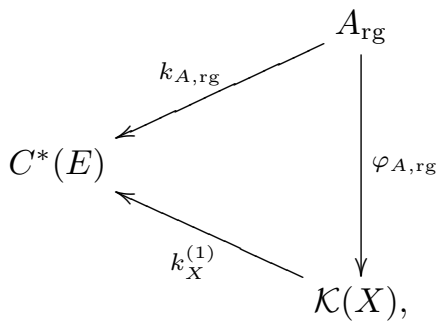

and it follows from $A$-strict continuity that the right triangle of (5) commutes.

The bottom triangle. For $\xi, \eta \in Y$ we have

$$
\begin{array}{rlr}
\overline{k_{X}^{(1)}}\left(\mu^{(1)}\left(\theta_{\xi, \eta}\right)\right) & =\overline{k_{X}^{(1)}}\left(\mu(\xi) \mu(\eta)^{*}\right) & \\
& =\overline{k_{X}}(\mu(\xi)) \overline{k_{X}}(\mu(\eta))^{*} \quad \text { (by Corollary A.14) } \\
& =\tau(\xi) \tau(\eta)^{*} \\
& =\tau^{(1)}\left(\theta_{\xi, \eta}\right) .
\end{array}
$$

The outer square. We need to show that if $f \in C_{0}\left(F_{\mathrm{rg}}^{0}\right)$, then

$$
\overline{\varphi_{A, \mathrm{rg}}} \circ \nu_{\mathrm{rg}}^{\prime}(f)=\mu^{(1)} \circ \varphi_{B, \mathrm{rg}}(f) .
$$

As in the commutative diagram $\sqrt{6}$ we can show

$$
\overline{\phi_{A, \mathrm{rg}}} \circ \nu_{\mathrm{rg}}^{\prime}(f)=\overline{\phi_{A}} \circ \nu_{\mathrm{rg}}(f)=\overline{\phi_{A}}(\nu(f)) \text {. }
$$

Also, $\varphi_{B, \mathrm{rg}}(f)=\varphi_{B}(f)$. Thus we must show

$$
\overline{\varphi_{A}}(\nu(f))=\mu^{(1)}\left(\varphi_{B}(f)\right)
$$

Since $f \circ r \in C_{0}\left(F^{1}\right)$, we can approximate $f \circ r$ uniformly by a function $h \in$ $C_{c}\left(F^{1}\right)$, and by Kat04, Lemmas 1.15 and 1.16] there are $\xi_{i}, \eta_{i} \in C_{c}\left(F^{1}\right)$ for $1=$ $1 \ldots n$ such that

- $h=\sum_{1}^{n} \xi_{i} \overline{\eta_{i}}$;

- $\pi_{F}(h)=\sum_{1}^{n} \theta_{\xi_{i}, \eta_{i}}$;

- for each $i$ we have $\xi_{i}(e) \eta_{i}\left(e^{\prime}\right)=0$ for all $e \neq e^{\prime}$ in $F^{1}$ with $s(e)=s\left(e^{\prime}\right)$. 
For $\zeta \in C_{c}\left(E^{1}\right)$ and $e \in E^{1}$ we have

$$
\begin{aligned}
\left(\mu^{(1)}\left(\theta_{\xi_{i}, \eta_{i}}\right) \zeta\right)(e) & =\left(\mu\left(\xi_{i}\right) \mu\left(\eta_{i}\right)^{*} \zeta\right)(e) \\
& =\xi_{i}(q(e))\left(\mu\left(\eta_{i}\right)^{*} \zeta\right)(s(e)) \\
& =\xi_{i}(q(e)) \sum_{s\left(e^{\prime}\right)=s(e)} \overline{\eta_{i}\left(q\left(e^{\prime}\right)\right)} \zeta\left(e^{\prime}\right) \\
& =\xi_{i}(q(e)) \sum_{s\left(q\left(e^{\prime}\right)\right)=s(q(e))} \overline{\eta_{i}\left(q\left(e^{\prime}\right)\right)} \zeta\left(e^{\prime}\right) \\
& =\xi_{i}(q(e)) \overline{\eta_{i}(q(e))} \zeta(e) .
\end{aligned}
$$

Thus

$$
\begin{aligned}
\left(\mu^{(1)}\left(\pi_{F}(h) \zeta\right)(e)\right. & =\sum_{1}^{n} \xi_{i}(q(e)) \overline{\eta_{i}(q(e))} \zeta(e) \\
& =h(q(e)) \zeta(e) .
\end{aligned}
$$

By our choice of $h$ we have

$$
\begin{aligned}
(\nu(f) \cdot \zeta)(e) & =\nu(f)(r(e)) \zeta(e) \\
& =f \circ q(r(e)) \zeta(e) \\
& =f \circ r(q(e)) \zeta(e) \\
& \approx h(q(e)) \zeta(e) .
\end{aligned}
$$

Let $K:=\operatorname{supp} \zeta$. The above approximation is uniform over $\zeta$ in the subspace

$$
C_{K}\left(E^{1}\right):=\left\{\xi \in C_{c}\left(E^{1}\right): \operatorname{supp} \xi \subset K\right\},
$$

so by the elementary Lemma 5.7 below we have

$$
\nu(f) \cdot \zeta \approx \mu^{(1)}\left(\pi_{F}(h)\right) \zeta \quad \text { in } X .
$$

Now, by definition we have

$$
\nu(f) \cdot \zeta=\overline{\varphi_{A}}(\nu(f)) \zeta
$$

so we get

$$
\overline{\varphi_{A}}(\nu(f)) \zeta \approx \mu^{(1)}\left(\pi_{F}(h)\right) \zeta
$$

Since $f \circ r \approx h$, we have $\varphi_{B}(f) \approx \pi_{F}(h)$, so

$$
\begin{aligned}
\mu^{(1)}\left(\varphi_{B}(f)\right) \zeta & \approx \mu^{(1)}\left(\pi_{F}(h)\right) \zeta \\
& \approx \overline{\varphi_{A}}(\nu(f)) \zeta .
\end{aligned}
$$

Thus $\left\|\mu^{(1)}\left(\varphi_{B}(f)\right) \zeta-\overline{\varphi_{A}}(\nu(f)) \zeta\right\|$ is arbitrarily small, so we must have

$$
\mu^{(1)}\left(\varphi_{B}(f)\right) \zeta=\overline{\varphi_{A}}(\nu(f)) \zeta
$$

Since $\zeta$ was an arbitrary element of $C_{c}\left(E^{1}\right)$, which is dense in $X$, we have shown that the outer square commutes.

In the above argument we used the following lemma:

Lemma 5.7. For any compact subset $K \subset E^{1}$, on the subspace $C_{K}\left(E^{1}\right)$ of $X$ the Hilbert-module norm from $X$ is equivalent to the uniform norm. 
Proof. The uniform norm on $C_{K}\left(E^{1}\right)$ is of course smaller than the Hilbert-module norm from $X$. On the other hand, by Lemma 3.9 (ii) we can let $d$ be an upper bound for the cardinalities of the intersections $K \cap s^{-1}(v)$ for $v \in E^{0}$. Then for any $v \in E^{0}$ we have

$$
\sum_{s(e)=v}|\xi(e)|^{2} \leq d\|\xi\|_{u}^{2}
$$

where $\|\cdot\|_{u}$ denotes the uniform norm.

It now follows that the left triangle of (5) commutes, and hence that the Toeplitz representation $(\tau, \pi): Y \rightarrow M\left(C^{*}(E)\right)$ is Cuntz-Pimsner covariant.

Step 4. To see that $\tau \times \pi: C^{*}(F) \rightarrow M\left(C^{*}(E)\right)$ is injective, we apply the GaugeInvariant Uniqueness Theorem [Kat04, Theorem 4.5]. For this we need to show that:

(1) $\pi: B \rightarrow M\left(C^{*}(E)\right)$ is injective, and

(2) for all $z \in \mathbb{T}$ there is an automorphism $\sigma_{z}$ of $(\tau \times \pi)\left(C^{*}(F)\right)$ such that $\sigma_{z}(\tau(\xi))=z \tau(\xi)$ for $\xi \in C_{c}\left(F^{1}\right)$ and $\sigma_{z}(\pi(f))=\pi(f)$ for $f \in C_{c}\left(F^{0}\right)$.

For (1), just note that both $\nu: B \rightarrow M(A)$ and $\overline{k_{A}}: M(A) \rightarrow M\left(C^{*}(E)\right)$ are injective (the latter because $k_{A}: A \rightarrow C^{*}(E)$ is), and hence so is $\overline{k_{A}} \circ \nu$.

For (2), we extend the gauge automorphism $\gamma_{z}$ of $C^{*}(E)$ to an automorphism $\overline{\gamma_{z}}$ of the multiplier algebra $M\left(C^{*}(E)\right)$, and note that by strict and $A$-strict continuity we have

$$
\begin{aligned}
& -\overline{\gamma_{z}} \circ \overline{k_{X}}=z \overline{k_{X}} \text { on } M_{A}(X), \text { and } \\
& \text { - } \overline{\gamma_{z}} \circ \overline{k_{A}}=\overline{k_{A}} \text { on } M(A) \text {. }
\end{aligned}
$$

Step 5. Finally, we need to show that the image $(\tau \times \pi)\left(C^{*}(F)\right)$ coincides with the generalized fixed-point algebra of $C^{*}(E)$ under the action $\alpha$ of $G$. We have an action $\beta$ of $G$ on $C_{0}\left(E^{0}\right)$ corresponding to the free and proper action on $E^{0}$, and a $G$-equivariant nondegenerate homomorphism $k_{A}: A=C_{0}\left(E^{0}\right) \rightarrow C^{*}(E) \subset$ $M\left(C^{*}(E)\right)$, so we can form the generalized fixed-point algebra

$$
C^{*}(E)^{\alpha}=\operatorname{Fix}\left(C^{*}(E), \alpha, k_{A}\right),
$$

and we will show that it coincides with $(\tau \times \pi)\left(C^{*}(F)\right)$. We begin with the inclusion

$$
\operatorname{Fix}\left(C^{*}(E), \alpha, k_{A}\right) \subset(\tau \times \pi)\left(C^{*}(F)\right),
$$

which will occupy us for some time.

For $n \in \mathbb{N}=\{0,1,2, \ldots\}$ let $E^{n}$ denote the space of paths in $E$ of length $n$ (with the relative product topology). Then by Kat04, $E^{n}$ is naturally a topological graph with vertex space $E^{0}$, and the associated $A$-correspondence $\mathcal{H}\left(E^{n}\right)$ is isomorphic to the $n$-fold balanced tensor product

$$
X \otimes_{A} \cdots \otimes_{A} X
$$

which we denote simply by $X^{n}$. We let $k_{X}^{n}: X^{n} \rightarrow C^{*}(E)$ be the representation corresponding to the $n$-fold tensor power of $k_{X}$. Then

$$
C^{*}(E)=\overline{\operatorname{span}}\left\{k_{X}^{n}\left(C_{c}\left(E^{n}\right)\right) k_{X}^{m}\left(C_{c}\left(E^{m}\right)\right)^{*}: n, m \in \mathbb{N}\right\} .
$$

Our hypotheses on $E$, namely that $G$ acts freely and properly, carry over to $E^{n}$. We use the same notational conventions as for the quotient graph $F=E / G$, and we write $\tau^{n}=\overline{k_{X}^{n}} \circ \mu$. 
Now let $f, h \in C_{c}\left(E^{0}\right)$ and $a \in C^{*}(E)$. We must show that

$$
\Phi\left(k_{A}(f) a k_{A}(h)\right) \in(\tau \times \pi)\left(C^{*}(F)\right) .
$$

We may assume that

$$
a=k_{X}^{n}(\xi) k_{Y}^{m}(\eta)^{*},
$$

where $\xi \in C_{c}\left(E^{n}\right)$ and $\eta \in C_{c}\left(E^{m}\right)$. By the elementary Lemma 5.8 below, we can find finitely many functions $\xi_{i} \in C_{c}\left(F^{n}\right), \eta_{j} \in C_{c}\left(F^{m}\right), f_{i}, g_{j} \in C_{c}\left(E^{0}\right)$ such that

$$
f \cdot \xi \approx \sum_{i} \mu\left(\xi_{i}\right) \cdot f_{i} \quad \text { and } \quad \eta \approx \sum_{j} g_{j} \cdot \mu\left(\eta_{j}\right) .
$$

Then for each $i, j$, again by Lemma 5.8 there exist finitely many $f_{i j l} \in C_{c}\left(E^{0}\right), \eta_{i j l} \in$ $C_{c}\left(F^{m}\right)$ such that

$$
\mu\left(\eta_{j}\right) \cdot \overline{f_{i}} \approx \sum_{l} f_{i j l} \cdot \mu\left(\eta_{i j l}\right)
$$

We have

$$
\begin{aligned}
k_{A}(f) a & =k_{A}(f) k_{X}^{n}(\xi) k_{X}^{m}(\eta)^{*} \\
& =k_{X}^{n}(f \cdot \xi) k_{X}^{m}(\eta)^{*} \\
& \approx \sum_{i j} k_{X}^{n}\left(\mu\left(\xi_{i}\right) \cdot f_{i}\right) k_{X}^{m}\left(g_{j} \cdot \mu\left(\eta_{j}\right)\right)^{*} \\
& =\sum_{i j} \overline{k_{X}^{n}}\left(\mu\left(\xi_{i}\right)\right) k_{A}\left(f_{i}\right) \overline{k_{X}^{m}}\left(\mu\left(\eta_{j}\right)\right)^{*} k_{A}\left(\overline{g_{j}}\right) \\
& =\sum_{i j} \overline{k_{X}^{n}}\left(\mu\left(\xi_{i}\right)\right)\left(\overline{k_{X}^{m}}\left(\mu\left(\eta_{j}\right)\right) k_{A}\left(\overline{f_{i}}\right)\right)^{*} k_{A}\left(\overline{g_{j}}\right) \\
& =\sum_{i j} \overline{k_{X}^{n}}\left(\mu\left(\xi_{i}\right)\right)\left(k_{X}^{m}\left(\mu\left(\eta_{j}\right) \cdot \overline{f_{i}}\right)\right)^{*} k_{A}\left(\overline{g_{j}}\right) \\
& \approx \sum_{i j l} \overline{k_{X}^{n}}\left(\mu\left(\xi_{i}\right)\right) k_{X}^{m}\left(f_{i j l} \cdot \mu\left(\eta_{i j l}\right)\right)^{*} k_{A}\left(\overline{g_{j}}\right) \\
& =\sum_{i j l} \overline{k_{X}^{n}}\left(\mu\left(\xi_{i}\right)\right) \tau^{m}\left(\eta_{i j l}\right)^{*} k_{A}\left(\overline{f_{i j l} g_{j}}\right) .
\end{aligned}
$$

Now, we can choose $h^{\prime} \in C_{c}\left(E^{0}\right)$ such that

$$
h^{\prime} f=f \quad \text { and } \quad h^{\prime} \cdot \mu\left(\xi_{i}\right)=\mu\left(\xi_{i}\right) \quad \text { for all } \quad i .
$$

Then we have

$$
\begin{aligned}
\Phi\left(k_{A}(f) a k_{A}(h)\right) & =\Phi\left(k_{A}\left(h^{\prime}\right) k_{A}(f) a k_{A}(h)\right) \\
& \approx \sum_{i j l} \Phi\left(k_{A}\left(h^{\prime}\right) \overline{k_{X}^{n}}\left(\mu\left(\xi_{i}\right)\right) \tau^{m}\left(\eta_{i j l}\right)^{*} k_{A}\left(\overline{f_{i j l} g_{j}}\right)\right) \\
& =\sum_{i j l} \Phi\left(\overline{k_{X}^{n}}\left(\mu\left(\xi_{i}\right)\right) \tau^{m}\left(\eta_{i j l}\right)^{*} k_{A}\left(\overline{f_{i j l} g_{j}}\right)\right) \\
& =\sum_{i j l} \tau^{n}\left(\xi_{i}\right) \tau^{m}\left(\eta_{i j l}\right)^{*} \Phi\left(k_{A}\left(\overline{f_{i j l} g_{j}}\right)\right) .
\end{aligned}
$$


Since $\tau^{n}\left(\xi_{i}\right) \tau^{m}\left(\eta_{i j l}\right)^{*} \in(\tau \times \pi)\left(C^{*}(F)\right)$ and

$$
\begin{aligned}
\Phi \circ k_{A}\left(C_{c}\left(E^{0}\right)\right) & =\overline{k_{A}} \circ \Phi^{\beta}\left(C_{c}\left(E^{0}\right)\right) \\
& \subset \overline{k_{A}} \circ \nu\left(C_{c}\left(F^{0}\right)\right) \\
& =\pi\left(C_{c}\left(F^{0}\right)\right) \\
& \subset(\tau \times \pi)\left(C^{*}(F)\right),
\end{aligned}
$$

it follows that $\Phi\left(k_{A}(f) a k_{A}(h)\right) \in(\tau \times \pi)\left(C^{*}(F)\right)$.

The above argument used the following elementary lemma:

Lemma 5.8. In the norm of $X$,

$$
C_{c}\left(E^{1}\right) \subset \overline{\operatorname{span}}\left\{\mu\left(C_{c}\left(F^{1}\right)\right) \cdot C_{c}\left(E^{0}\right)\right\} \cap \overline{\operatorname{span}}\left\{C_{c}\left(E^{0}\right) \cdot \mu\left(C_{c}\left(F^{1}\right)\right)\right\} .
$$

Proof. By Theorem 3.4 we may assume that $E^{1}=s^{*}\left(E^{0}\right)$. Let $\theta: s^{*}\left(E^{0}\right) \rightarrow r^{*}\left(E^{0}\right)$ be the isomorphism of Theorem 3.4 . Note that

$$
\mu(\eta) \cdot f=\left.(\eta \otimes f)\right|_{s^{*}\left(E^{0}\right)} \quad \text { and } \quad f \cdot \mu(\eta)=(\eta \otimes f) \circ \theta,
$$

where $(\eta \otimes f)(e, v)=\eta(e) f(v)$ for $(e, v) \in F^{1} \times E^{0}$.

Let $\xi \in C_{c}\left(E^{1}\right)$. Then there is $\xi^{\prime} \in C_{c}\left(F^{1} \times E^{0}\right)$ such that

$$
\xi=\left.\xi^{\prime}\right|_{s^{*}\left(E^{0}\right)},
$$

because $s^{*}\left(E^{0}\right)$ is a closed subset of $F^{1} \times E^{0}$. Then there are finitely many $\eta_{i} \in$ $C_{c}\left(F^{1}\right), f_{i} \in C_{c}\left(E^{0}\right)$ such that

$$
\xi^{\prime} \approx \sum_{i}\left(\eta_{i} \otimes f_{i}\right)
$$

Thus

$$
\left.\xi \approx \sum_{i}\left(\eta_{i} \otimes f_{i}\right)\right|_{s^{*}\left(E^{0}\right)}=\sum_{i} \mu\left(\eta_{i}\right) \cdot f_{i} .
$$

This shows the inclusion in the first set. For the other one, since $\xi \circ \theta^{-1} \in$ $C_{c}\left(r^{*}\left(E^{0}\right)\right)$, there is $\zeta \in C_{c}\left(F^{1} \times E^{0}\right)$ such that

$$
\xi \circ \theta^{-1}=\left.\zeta\right|_{r^{*}\left(E^{0}\right)},
$$

and then as above we can approximate

$$
\zeta \approx \sum_{i}\left(\eta_{i} \otimes f_{i}\right)
$$

so that

$$
\left.\xi \circ \theta^{-1} \approx \sum_{i}\left(\eta_{i} \otimes f_{i}\right)\right|_{r^{*}\left(E^{0}\right)},
$$

and hence

$$
\xi \approx \sum_{i}\left(\eta_{i} \otimes f_{i}\right) \circ \theta=\sum_{i} f_{i} \cdot \mu\left(\eta_{i}\right)
$$

We have shown that $\operatorname{Fix}\left(C^{*}(E), \alpha, k_{A}\right) \subset(\tau \times \pi)\left(C^{*}(F)\right)$, and we turn to the opposite inclusion. We need the following easy lemma.

Lemma 5.9. For all $f \in C_{c}\left(F^{0}\right)$ there exists $h \in C_{c}\left(E^{0}\right)$ such that $\nu(f)=\Phi^{\beta}(h)$. 
Proof. Put $M=\operatorname{supp} f$, a compact subset of $F^{0}$. Since the quotient map $q: E^{0} \rightarrow$ $F^{0}$ is a continuous open surjection, it is a standard fact that there exists a compact set $K \subset E^{0}$ such that $q(K)=M$. Choose $h_{1} \in C_{c}\left(E^{0}\right)$ such that $h_{1}(v) \neq 0$ for all $v \in K$. Then $\Phi^{\beta}\left(h_{1}\right) \neq 0$ on $q^{-1}(M)$, and there is a unique $h_{2} \in C_{c}\left(F^{0}\right)$ such that $\Phi^{\beta}\left(h_{1}\right)=\nu\left(h_{2}\right)$. Then $h_{2} \neq 0$ on $M$, so there exists $h_{3} \in C_{c}\left(F^{0}\right)$ such that

$$
h_{3}=\frac{f}{h_{2}} \quad \text { on } \quad M
$$

Then $f=h_{2} h_{3}$, so

$$
\begin{aligned}
\nu(f) & =\nu\left(h_{2}\right) \nu\left(h_{3}\right) \\
& =\Phi^{\beta}\left(h_{1}\right) \nu\left(h_{3}\right) \\
& =\Phi^{\beta}\left(h_{1} \nu\left(h_{3}\right)\right),
\end{aligned}
$$

so we can take $h=h_{1} \nu\left(h_{3}\right)$.

To finish, it suffices to show that $\tau(\xi)$ and $\pi(f)$ are in $\operatorname{Fix}\left(C^{*}(E), \alpha, k_{A}\right)$ for all $\xi \in C_{c}\left(F^{1}\right)$ and $f \in C_{c}\left(F^{0}\right)$. For $\xi$, choose $h \in C_{c}\left(F^{0}\right)$ such that $\xi=\xi \cdot h$. By Lemma 5.9 we can choose $h_{1} \in C_{c}\left(E^{0}\right)$ such that $\nu(h)=\Phi^{\beta}\left(h_{1}\right)$. We have

$$
\begin{aligned}
\tau(\xi) & =\tau(\xi \cdot h) \\
& =\tau(\xi) \pi(h) \\
& =\tau(\xi) \overline{k_{A}}(\nu(h)) \\
& =\tau(\xi) \overline{k_{A}}\left(\Phi^{\beta}\left(h_{1}\right)\right) \\
& =\tau(\xi) \Phi\left(k_{A}\left(h_{1}\right)\right) \\
& =\Phi\left(\tau(\xi) k_{A}\left(h_{1}\right)\right) \\
& =\Phi\left(\overline{k_{X}}(\mu(\xi)) k_{A}\left(h_{1}\right)\right) \\
& =\Phi\left(k_{X}\left(\mu(\xi) \cdot h_{1}\right)\right) .
\end{aligned}
$$

Now, by Corollary 3.9 we have $\mu(\xi) \cdot h_{1} \in C_{c}\left(E^{1}\right)$, so we can choose $h_{2} \in C_{c}\left(E^{0}\right)$ such that

$$
\mu(\xi) \cdot h_{1}=h_{2} \cdot \mu(\xi) \cdot h_{1},
$$

and then we can factor $h_{2}=h_{3} h_{4}$ with $h_{3}, h_{4} \in C_{c}\left(E^{0}\right)$. Then

$$
\begin{aligned}
\tau(\xi) & =\Phi\left(k_{X}\left(h_{3} h_{4} \cdot \mu(\xi) \cdot h_{1}\right)\right) \\
& =\Phi\left(k_{A}\left(h_{3}\right) k_{X}\left(h_{4} \cdot \mu(\xi)\right) k_{A}\left(h_{1}\right)\right) .
\end{aligned}
$$

Since $h_{4} \cdot \mu(\xi) \in C_{c}\left(E^{1}\right)$ (again by Corollary 3.9 , we have

$$
k_{X}\left(h_{4} \cdot \mu(\xi)\right) \in C^{*}(E),
$$

and therefore $\tau(\xi) \in \operatorname{Fix}\left(C^{*}(E), \alpha, k_{A}\right)$.

Our strategy for $f \in C_{c}\left(F^{0}\right)$ is similar, but things are somewhat easier this time: factor $f=f h_{1}$ with $h_{1} \in C_{c}\left(F^{0}\right)$, then choose $h_{2} \in C_{c}\left(E^{0}\right)$ such that 
$\nu\left(h_{1}\right)=\Phi^{\beta}\left(h_{2}\right)$, then factor $h_{2}=h_{3} h_{4} h_{5}$ with $h_{i} \in C_{c}\left(E^{0}\right)$. We have

$$
\begin{aligned}
\pi(f) & =\pi\left(f h_{1}\right) \\
& =\pi(f) \pi\left(h_{1}\right) \\
& =\pi(f) \overline{k_{A}}\left(\nu\left(h_{1}\right)\right) \\
& =\pi(f) \overline{k_{A}}\left(\Phi^{\beta}\left(h_{2}\right)\right) \\
& =\pi(f) \Phi\left(k_{A}\left(h_{3} h_{4} h_{5}\right)\right) \\
& =\Phi\left(\pi(f) k_{A}\left(h_{3} h_{4} h_{5}\right)\right) \\
& =\Phi\left(\overline{k_{A}}(\nu(f)) k_{A}\left(h_{3} h_{4} h_{5}\right)\right) \\
& =\Phi\left(k_{A}\left(h_{3}\right) k_{A}\left(f h_{3}\right) k_{A}\left(h_{5}\right)\right),
\end{aligned}
$$

which is in $\operatorname{Fix}\left(C^{*}(E), \alpha, k_{A}\right)$ because $k_{A}\left(f h_{3}\right) \in C^{*}(E)$.

Let $E$ be a topological graph and let $c: E^{1} \rightarrow G$ be a continuous map. Recall that $G$ acts freely and properly on $G \times{ }_{c} E$. Since $\left(G \times{ }_{c} E\right) / G \cong E$, we have by Theorem 5.6 .

Corollary 5.10. The $C^{*}$-algebras $C^{*}\left(E \times_{c} G\right) \rtimes_{r} G$ and $C^{*}(E)$ are strongly Morita equivalent. In particular, for a finitely generated locally compact group $G$ with generators $S=\left\{h_{1}, h_{2}, \ldots, h_{n}\right\}$ and Cayley graph $E(G, S)$, we get that $C^{*}(E(G, S)) \rtimes_{r} G$ is strongly Morita equivalent to the Cuntz algebra $\mathcal{O}_{n}$.

Remark 5.11. If $G$ is abelian, there is an action $\alpha^{c}$ of $\hat{G}$ on $C^{*}(E)$ such that

$$
\left(\alpha_{\chi}^{c} \xi\right)(e)=\langle\chi, c(e)\rangle \xi(e)
$$

for $\xi \in C_{c}\left(E^{1}\right)$ and $\chi \in \hat{G}$. Then

$$
C^{*}(E) \rtimes_{\alpha^{c}} \hat{G} \cong C^{*}\left(E \times_{c} G\right) .
$$

For $G$ non-abelian, we need to use the notion of coaction. This will be investigated in KQR10.

\section{ApPendix A. MULTIPLIERS OF CORRESPONDENCES}

In Section 5 we make extensive use of the multiplier bimodules of EKQR06, Chapter 1]. The bimodules in EKQR06 were called right-Hilbert $A-B$ bimodules, which are also commonly referred to as $A-B$ correspondences, where $A$ and $B$ are $C^{*}$-algebras. In most of this paper we are interested in correspondences over a single $C^{*}$-algebra, but occasionally we need the added generality of allowing $A$ and $B$ to be possibly different. Warning: there is a nondegeneracy issue; in the literature, $A-B$ correspondences are often (usually?) allowed to be degenerate, in the sense that the closed span of $A \cdot X$ might be smaller than $X$, but in EKQR06 the right-Hilbert bimodules are required to be nondegenerate. This will cause no problem here, though, because the correspondences associated to topological graphs are all nondegenerate, so that the results of EKQR06 may be applied freely.

To emphasize, throughout this appendix we will assume that all our correspondences are nondegenerate.

$A$ and $B$ are the coefficient algebras of the correspondence $X$, and we will sometimes denote the correspondence by ${ }_{A} X_{B}$ to indicate what the coefficient algebras are. Note that a Hilbert $B$-module is also a $\mathbb{C}-B$ correspondence. 
We recall briefly the theory of multiplier bimodules; see [EKQR06, Chapter 1] for more details. A multiplier of ${ }_{A} X_{B}$ is by definition an adjointable map from $B$ to $X$, and $M(X)$ denotes the set of all multipliers of $X$. Thus by definition we have $M(X)=\mathcal{L}(B, X)$. Note that the definition of $M(X)$ does not involve the left $A$-module structure at all; it is, in essence, a Hilbert $B$-module concept. However, $M(X)$ is an $\mathcal{L}(X)-M(B)$ correspondence, hence an $M(A)-M(B)$ correspondence because of our assumption that $X$ is nondegenerate as a left $A$ module. The restriction of the right $M(B)$-module action to $B$ is just the evaluation of adjointable maps; i.e., if $m \in M(X)$ and $b \in B$ then we write $m \cdot b$ to mean the value of the adjointable map $m: B \rightarrow X$ at the element $b$ of its domain. There is an embedding of $X$ in $M(X)$ such that the adjointable map associated to $\xi \in X$ is

$$
b \mapsto \xi \cdot b \quad \text { for } \quad b \in B .
$$

$M(X)$ has both an $\mathcal{L}(X)$-valued inner product

$$
\mathcal{L}(X)\langle m, n\rangle=m n^{*} \quad \text { for } \quad m, n \in M(X)
$$

and an $M(B)$-valued inner product

$$
\langle m, n\rangle_{M(B)}=\langle m, n\rangle=m^{*} n .
$$

If either $m$ or $n$ lies in $X$, then

$$
\mathcal{L}(X)\langle m, n\rangle \in \mathcal{K}(X) \quad \text { and } \quad\langle m, n\rangle \in B
$$

because, more generally, composing an adjointable operator on either side with a compact operator gives a compact operator. The restriction of the left inner product $\mathcal{L}(X)\langle\cdot, \cdot\rangle$ to $X$ gives the rank-one operators:

$$
\mathcal{L}(X)\langle\xi, \eta\rangle={ }_{\mathcal{K}(X)}\langle\xi, \eta\rangle=\theta_{\xi, \eta},
$$

and the restriction of the right inner product to $X$ is the given one. The left and right module actions of multipliers of the coefficient algebras $A$ and $B$, respectively, on an element $m \in M(X)$ are as follows: if $n \in M(A)$ then $n \cdot m$ is the element of $M(X)$ defined by

$$
(n \cdot m) \cdot b=n \cdot(m \cdot b) \quad \text { for } \quad b \in B
$$

and if $n \in M(B)$ then $m \cdot n$ is defined by

$$
(m \cdot n) \cdot b=m \cdot(n b) \quad \text { for } \quad b \in B .
$$

Of course, if $n \in B$ then $m \cdot n \in X$.

The strict topology on $M(X)$ is generated by the seminorms

$$
m \mapsto\|T \cdot m\| \quad \text { and } \quad m \mapsto\|m \cdot b\| \quad \text { for } \quad T \in \mathcal{K}(X), b \in B .
$$

The operations on the $M(A)-M(B)$ correspondence $M(X)$ are separately strictly continuous, and $M(X)$ is the strict completion of $X$.

Given another correspondence ${ }_{C} Y_{D}$ and homomorphisms $\pi: A \rightarrow M(C)$ and $\theta: B \rightarrow M(D)$, a linear map $\psi: X \rightarrow M(Y)$ is a $\pi-\theta$ compatible correspondence homomorphism if the two conditions

$$
\begin{aligned}
\langle\psi(\xi), \psi(\eta)\rangle_{M(D)} & =\theta\left(\langle\xi, \eta\rangle_{B}\right) \\
\psi(a \cdot \xi) & =\pi(a) \cdot \psi(\xi)
\end{aligned}
$$

hold. Note that $\psi$ will automatically satisfy

$$
\psi(\xi \cdot b)=\psi(\xi) \theta(b) .
$$


$\pi$ and $\theta$ are the coefficient homomorphisms of $\psi$, and sometimes we write ${ }_{\pi} \psi_{\theta}$ for the correspondence homomorphism to indicate what the coefficient homomorphisms are.

A correspondence homomorphism ${ }_{\pi} \psi_{\theta}$ is nondegenerate if $\pi$ and $\theta$ are nondegenerate and

$$
\overline{\operatorname{span}}\{\psi(X) \cdot D\}=Y,
$$

in which case ${ }_{\pi} \psi_{\theta}$ extends uniquely to a strictly continuous homomorphism

$$
\bar{\pi} \bar{\psi}_{\bar{\theta}}:{ }_{M(A)} M(X)_{M(B)} \rightarrow{ }_{M(C)} M(Y)_{M(D)} .
$$

(In fact, as for $C^{*}$-algebras, the extension is unique as just a correspondence homomorphism, and is automatically strictly continuous, although we will not need this fact.) If $A=B, C=D$, and $\pi=\theta$ we will write

$$
(\psi, \pi)={ }_{\pi} \psi_{\theta},
$$

and if in addition $Y=C$ is a correspondence over itself in the canonical way, and if both $\psi$ and $\pi$ map into $C$ (and not just $M(C)$ ), then $(\psi, \pi)$ reduces to the familiar concept of a Toeplitz representation of the $A$-correspondence $X$ into $C$.

If

$$
{ }_{\pi} \psi_{\theta}:{ }_{A} X_{B} \rightarrow M\left({ }_{C} Y_{D}\right)
$$

is a nondegenerate homomorphism, then there is a unique nondegenerate homomorphism

$$
\psi^{(1)}: \mathcal{K}(X) \rightarrow M(\mathcal{K}(Y))=\mathcal{L}(Y)
$$

such that

$$
\psi^{(1)}\left(\theta_{\xi, \eta}\right)=\psi(\xi) \psi(\eta)^{*} \quad \text { for } \quad \xi, \eta \in X
$$

and then, as usual, by nondegeneracy $\psi^{(1)}$ extends uniquely to a homomorphism

$$
\overline{\psi^{(1)}}: \mathcal{L}(X) \rightarrow \mathcal{L}(Y) .
$$

Note that even if $(\psi, \pi)$ is degenerate, there is still a homomorphism $\psi^{(1)}: \mathcal{K}(X) \rightarrow$ $\mathcal{K}(M(Y)) \subset M(\mathcal{K}(Y))$, but then there may not be an extension $\overline{\psi^{(1)}}$ to $\mathcal{L}(X)$ (although we will define a partial extension, under additional hypotheses, in Corollary A.14.

$C$-multipliers. Unfortunately, we must deal with possibly degenerate homomorphisms of correspondences, and for this reason we have been lead to develop a generalization of the " $C$-multipliers" of EKQR06, Section 1.4].

Example A.1. We can see why degeneracy is an issue for us already in the case of (discrete) directed graphs: let $E$ be the directed graph with a single non-loop edge, i.e.,

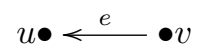

We have:

- $C^{*}(E)=M_{2}$;

- $X=\mathcal{H}(E)=\mathbb{C} \cdot \chi_{\{e\}}$;

- $k_{X}\left(\chi_{\{e\}}\right)=\left(\begin{array}{ll}0 & 1 \\ 0 & 0\end{array}\right)$;

- $k_{X}(X) C^{*}(E)=\left(\begin{array}{cc}* & * \\ 0 & 0\end{array}\right)$,

so the canonical Toeplitz representation $\left(k_{X}, k_{A}\right)$ is degenerate (where $A=\mathbb{C}^{2}$ ). 
Remark A.2. Of course, there are situations in which degeneracy is not an issue; for example, if the correspondence $X$ is actually an imprimitivity bimodule (so that $\varphi_{A}: A \rightarrow \mathcal{K}(X)$ is an isomorphism), then every Cuntz-Pimsner covariant Toeplitz representation $(\psi, \pi)$ of $X$ to a $C^{*}$-algebra $B$ for which $\pi$ is nondegenerate is automatically nondegenerate as a correspondence homomorphism, i.e., $\psi(X) B=$ $B$, by [KQR97, Lemma 5.1]. Note also that whenever $E$ is a topological graph with $s$ and $r$ both surjective and $r$ proper, then $k_{X}: X \rightarrow C^{*}(E)$ will be nondegenerate, hence will extend uniquely to the multiplier bimodule $M(X)$.

The following is similar to that in EKQR06. To prepare for the general framework introduced below, we begin with some prefatory comments. First, to establish context, we recall that the " $C$-multipliers" in EKQR06 involved tensor products: if $A$ and $C$ are $C^{*}$-algebras, then [EKQR06] defined $M_{C}(A \otimes C)$ to be all multipliers of $A \otimes C$ that multiply $1 \otimes C$ into $A \otimes C$. Here we need a generalization to situations where there are no tensor products.

Definition A.3. Let $\kappa: C \rightarrow M(A)$ be a nondegenerate homomorphism.

(1) A $C$-multiplier of $A$ is a multiplier $m \in M(A)$ such that

$$
\kappa(C) m \cup m \kappa(C) \subset A,
$$

and $M_{C}(A)$ denotes the set of all $C$-multipliers of $A$.

(2) The $C$-strict topology on $M_{C}(A)$ is generated by the seminorms

$$
m \mapsto\|\kappa(c) m\| \quad \text { and } \quad \mapsto\|m \kappa(c)\| \text { for } \quad c \in C .
$$

Often $C$ will be a nondegenerate $C^{*}$-subalgebra of $M(A)$ and $\kappa$ will be the inclusion map.

The following generalizes EKQR06, Proposition A.5]:

\section{Lemma A.4.}

(1) The $C$-strict topology on $M_{C}(A)$ is stronger than the relative strict topology from $M(A)$.

(2) $M_{C}(A)$ is a $C^{*}$-subalgebra of $M(A)$, and multiplication and involution are separately $C$-strictly continuous on $M_{C}(A)$.

(3) $M_{C}(A)$ is the $C$-strict completion of $A$;

(4) $M_{C}(A)$ is an $M(C)$-subbimodule of $M(A)$.

Proof. (1) Let $m_{i} \rightarrow 0 C$-strictly in $M_{C}(A)$. We must show that if $a \in A$ then $\left\|a m_{i}\right\|$ and $\left\|m_{i} a\right\|$ both tend to 0 . By the Hewitt-Cohen factorization theorem (see, for example, RW98, Proposition 2.33]) we can factor $a=a^{\prime} \kappa(c)$ for some $a^{\prime} \in A$ and $c \in C$, and then

$$
\left\|a m_{i}\right\|=\left\|a^{\prime} \kappa(c) m_{i}\right\| \leq\left\|a^{\prime}\right\|\left\|\kappa(c) m_{i}\right\| \rightarrow 0,
$$

and similarly for $m_{i} a$.

(2) It follows straight from the definitions that $M_{C}(A)$ is a $C^{*}$-subalgebra. Let $m_{i} \rightarrow 0 C$-strictly in $M_{C}(A)$, and let $n \in M(A)$. We must show that $m_{i} n \rightarrow 0$ $C$-strictly in $M_{C}(A)$, and it will follow similarly that $n m_{i} \rightarrow 0 C$-strictly. For $c \in C$ we have

$$
\left\|\kappa(c) m_{i} n\right\| \leq\left\|\kappa(c) m_{i}\right\|\|n\| \rightarrow 0
$$

because $m_{i} \rightarrow 0 C$-strictly, and

$$
\left\|m_{i} n \kappa(c)\right\| \rightarrow 0
$$


because $n \kappa(c) \in A$ and $m_{i} \rightarrow 0$ strictly by (1).

It is even easier to verify that $m_{i}^{*} \rightarrow 0 C$-strictly.

(3) We first show that $A$ is $C$-strictly dense in $M_{C}(A)$. Let $m \in M_{C}(A)$, and let $\left\{e_{i}\right\}$ be an approximate identity for $C$. We will show that $\kappa\left(e_{i}\right) m \rightarrow m C$-strictly. For $c \in C$ we have

$$
\left\|\kappa(c)\left(\kappa\left(e_{i}\right) m-m\right)\right\|=\left\|\kappa\left(c e_{i}-c\right) \cdot m\right\| \leq\left\|c e_{i}-c\right\|\|m\| \rightarrow 0
$$

while

$$
\left\|\left(\kappa\left(e_{i}\right) m-m\right) \kappa(c)\right\|=\left\|\kappa\left(e_{i}\right) m \kappa(c)-m \kappa(c)\right\| \rightarrow 0
$$

because $\kappa\left(e_{i}\right) \rightarrow 0$ strictly in $M(A)$.

Now we verify the $C$-strict completeness. Let $\left\{m_{i}\right\}$ be a $C$-strictly Cauchy net in $M_{C}(A)$. Then it is also strictly Cauchy, so converges strictly to some $m \in M(A)$ by strict completeness. We will show that $m \in M_{C}(A)$ and $m_{i} \rightarrow m C$-strictly. Let $\epsilon>0$ and $c \in C$. Choose $i_{0}$ such that

$$
\left\|\kappa(c)\left(m_{i}-m_{j}\right)\right\| \leq \epsilon \quad \text { for all } i, j \geq i_{0} .
$$

Then for all $a \in A$ with $\|a\| \leq 1$ we have $\left\|\left(m_{j}-m\right) a\right\| \rightarrow 0$, so for all $i \geq i_{0}$ we have

$$
\left\|\kappa(c)\left(m_{i}-m\right) a\right\|=\lim _{j}\left\|\kappa(c)\left(m_{i}-m_{j}\right) a\right\| \leq \limsup _{j}\left\|\kappa(c)\left(m_{i}-m_{j}\right)\right\| \leq \epsilon,
$$

and hence $\left\|\kappa(c)\left(m_{i}-m\right)\right\| \leq \epsilon$. It follows that $\left\|\kappa(c)\left(m_{i}-m\right)\right\| \rightarrow 0$. Since $\kappa(c) m_{i} \in$ $A$ for all $i$, we have $\kappa(c) m \in A$. Similarly, $\left\|\left(m_{i}-m\right) \kappa(c)\right\| \rightarrow 0$ and $m \kappa(c) \in A$. Thus $m \in M_{C}(A)$, and the above arguments then show that $m_{i} \rightarrow m C$-strictly.

(4) We have

$$
\bar{\kappa}(M(C)) M_{C}(A) \kappa(C) \subset \bar{\kappa}(M(C)) A \subset A
$$

and

$$
\kappa(C) \bar{\kappa}(M(C)) M_{C}(A) \subset \kappa(C) M_{C}(A) \subset A,
$$

so $\bar{\kappa}(M(C)) M_{C}(A) \subset M_{C}(A)$, and similarly for $M_{C}(A) \bar{\kappa}(M(C))$.

The whole point of $C$-multipliers is to extend degenerate homomorphisms. The following generalizes [EKQR06, Proposition A.6]:

Lemma A.5. Let $\kappa: C \rightarrow M(A), \sigma: D \rightarrow M(B), \pi: A \rightarrow M_{D}(B)$, and $\lambda: C \rightarrow M(\sigma(D))$ be homomorphisms, with $\kappa, \sigma$, and $\lambda$ nondegenerate, such that

$$
\pi(\kappa(c) a)=\lambda(c) \pi(a) \quad \text { for } \quad c \in C, a \in A .
$$

Then $\pi$ extends uniquely to a $C$-strict to D-strictly continuous homomorphism $\bar{\pi}$ : $M_{C}(A) \rightarrow M_{D}(B)$.

Moreover, for $n \in M(C)$ and $m \in M_{C}(A)$ we have

$$
\bar{\pi}(\bar{\kappa}(n) m)=\bar{\lambda}(n) \bar{\pi}(m) \quad \text { and } \quad \bar{\pi}(m \bar{\kappa}(n))=\bar{\pi}(m) \bar{\lambda}(n) .
$$

Note that, in the above lemma, $M(\sigma(D))$ is identified with the idealizer of $\sigma(D)$ in $M(B)$ (and this is valid since $\sigma$ is nondegenerate), and $\bar{\lambda}$ and $\bar{\kappa}$ denote the canonical extensions to the multiplier algebras (which exist by nondegeneracy). 
Proof. We first show that $\pi$ is $C$-strict to $D$-strictly continuous. Let $m_{i} \rightarrow 0 C$ strictly in $A$, and let $d \in D$. By the Hewitt-Cohen factorization theorem we can factor $\sigma(d)=\sigma\left(d^{\prime}\right) \lambda(c)$ for some $d^{\prime} \in D$ and $c \in C$, and then

$$
\begin{aligned}
\left\|\sigma(d) \pi\left(m_{i}\right)\right\| & =\left\|\sigma\left(d^{\prime}\right) \lambda(c) \pi\left(m_{i}\right)\right\| \\
& =\left\|\sigma\left(d^{\prime}\right) \pi\left(\kappa(c) m_{i}\right)\right\| \\
& \leq\left\|d^{\prime}\right\|\left\|\kappa(c) m_{i}\right\| \\
& \rightarrow 0,
\end{aligned}
$$

and similarly for $\pi\left(m_{i}\right) \sigma(d)$.

Thus, by completeness there is a unique $C$-strict to $D$-strictly continuous linear extension $\bar{\pi}: M_{C}(A) \rightarrow M_{D}(B)$. Since the algebraic operations on $M_{C}(A)$ and $M_{D}(B)$ are separately continuous for the $C$-strict and $D$-strict topologies, respectively, $\bar{\pi}$ is a $*$-homomorphism.

The other part follows from continuity and density.

Remark A.6. (1) In fact, the above extension $\bar{\pi}$ is unique as a homomorphism, but we will not need this.

(2) The above lemma could be the starting point for an investigation of what one might call "decorated $C^{*}$-algebras" (where $A$ is "decorated" by $C$ and $B$ is decorated by $D$ ). However, we will not pursue this any further here.

One of our applications of the above result is described by the following special case, in which we will actually need $\pi$ to take values in $B$ itself:

Corollary A.7. Let $I$ be an ideal of $A$, and let $\pi: A \rightarrow B$ be a nondegenerate homomorphism. Then the restriction $\pi_{I}$ of $\pi$ to I extends uniquely to an A-strictly continuous homomorphism $\overline{\pi_{I}}: M_{A}(I) \rightarrow M_{A}(B)$.

Proof. Apply Lemma A.5 with $A, I$ playing the role of $C, A$, with the canonical homomorphism $\rho: A \rightarrow M(I)$ playing the role of $\kappa$, with $D=A$ and $\sigma=\lambda=\pi$, and with $\pi_{I}$ playing the role of $\pi$. Since

$$
\pi_{I}(I) \subset B \subset M_{A}(B),
$$

the hypotheses of the lemma are satisfied.

$C$-multiplier bimodules. The preceding subsection was really only a prelude for the current one, where we generalize the $C$-multiplier bimodules of EKQR06, Section 1.4] for tensor products.

In addition to the preceding nondegenerate homomorphism $\kappa: C \rightarrow M(A)$, suppose that we also have a nondegenerate $A$-correspondence $X$, and let $\varphi_{A}$ : $A \rightarrow \mathcal{L}(X)$ be the associated homomorphism. Then we can compose to get a nondegenerate homomorphism

$$
\varphi_{C}:=\overline{\varphi_{A}} \circ \kappa: C \rightarrow \mathcal{L}(X),
$$

where $\overline{\varphi_{A}}$ denotes the canonical extension of $\varphi_{A}: A \rightarrow \mathcal{L}(X)$ to $M(A)$, so $X$ becomes a nondegenerate $C-A$ correspondence. Similarly on the right, so $X$ becomes a nondegenerate $C$-bimodule (but not a correspondence, because the $A$ valued inner product cannot be turned into a $C$-valued one). Since $M(X)$ is an $M(A)$-correspondence, by composing with $\bar{\kappa}$ we get an $M(C)$-bimodule structure on $M(X)$ (but again, not a correspondence). 
Definition A.8. Let $X$ be a nondegenerate $A$-correspondence, and let $\kappa: C \rightarrow$ $M(A)$ be a nondegenerate homomorphism.

(1) A $C$-multiplier of $X$ is a multiplier $m \in M(X)$ such that

$$
\kappa(C) \cdot m \cup m \cdot \kappa(C) \subset X,
$$

and $M_{C}(X)$ denotes the set of all $C$-multipliers of $X$.

(2) The $C$-strict topology on $M_{C}(X)$ is generated by the seminorms

$$
m \mapsto\|\kappa(c) \cdot m\| \quad \text { and } \quad m \mapsto\|m \cdot \kappa(c)\| \quad \text { for } \quad c \in C .
$$

The $C$-multiplier algebra $M_{C}(A)$ from the preceding subsection is the special case of $M_{C}(X)$ where $X=A$ regarded as an $A$-correspondence in the usual way.

The following generalizes EKQR06, Lemma 1.40]:

\section{Lemma A.9.}

(1) The $C$-strict topology is stronger than the relative strict topology on $M_{C}(X)$.

(2) $M_{C}(X)$ is an $M_{C}(A)$-correspondence with respect to the restrictions of the operations of the $M(A)$-correspondence $M(X)$, and the operations are separately $C$-strictly continuous.

(3) $\mathcal{K}\left(M_{C}(X)\right) \subset M_{C}(\mathcal{K}(X))$.

(4) $M_{C}(X)$ is the $C$-strict completion of $X$.

(5) $M_{C}(X)$ is an $M(C)$-subbimodule of $M(X)$.

Proof. Many of the arguments are quite similar to those in the proof of Lemma A.4.

(1) If $m_{i} \rightarrow 0 C$-strictly in $M_{C}(X)$, we must show that if $T \in \mathcal{K}(X)$ and $a \in A$ then both $\left\|T m_{i}\right\|$ and $\left\|m_{i} \cdot a\right\|$ tend to 0 , and the argument is similar to Lemma A.4.

(2) If $n \in M_{C}(A)$ and $m \in M_{C}(X)$ then $n \cdot m$ is in $M(X)$ because $M(X)$ is an $M(A)$-correspondence; we must show that it is in $M_{C}(X)$. For $c \in C$, note that $\kappa(c) n \in A$, so because $\kappa: C \rightarrow M(A)$ is nondegenerate we can factor $\kappa(c) n=a \kappa\left(c^{\prime}\right)$ with $a \in A$ and $c^{\prime} \in C$, and then we have

$$
\kappa(c) \cdot(n \cdot m)=(\kappa(c) n) \cdot m=\left(a \kappa\left(c^{\prime}\right)\right) \cdot m=a \cdot\left(\kappa\left(c^{\prime}\right) \cdot m\right) \in X
$$

because $\kappa\left(c^{\prime}\right) \cdot m \in X$.

On the other hand, we have

$$
(n \cdot m) \cdot \kappa(c)=n \cdot(m \cdot \kappa(c)) \in X
$$

because $m \cdot \kappa(c) \in X$ and $n \in M(A)$.

The $M(A)$-valued inner product on $M(X)$ restricts to one on $M_{C}(X)$; we must show that for $m, n \in M_{C}(X)$ the inner product $\langle m, n\rangle$ is in $M_{C}(A)$. For $c \in C$ we have

$$
\langle m, n\rangle \kappa(c)=\langle m, n \cdot \kappa(c)\rangle \in A,
$$

because $n \cdot \kappa(c) \in X$ and $\langle M(X), X\rangle \subset A$, and similarly $\kappa(c)\langle m, n\rangle \in A$.

The separate continuity is similar to Lemma A.4. For example, if $m_{i} \rightarrow 0 \mathrm{C}$ strictly in $M_{C}(X), n \in M_{C}(X)$, and $c \in C$, then

$$
\left\|\left\langle m_{i}, n\right\rangle \kappa(c)\right\|=\left\|\left\langle m_{i}, n \cdot \kappa(c)\right\rangle\right\| \rightarrow 0
$$

because $n \cdot \kappa(c) \in X, m_{i} \rightarrow 0$ strictly by (1), and the operations in $M(X)$ are separately strictly continuous, while

$$
\left\|\kappa(c)\left\langle m_{i}, n\right\rangle\right\|=\left\|\left\langle m_{i} \cdot \kappa\left(c^{*}\right), n\right\rangle\right\| \rightarrow 0
$$

because $\left\|m_{i} \cdot \kappa\left(c^{*}\right)\right\| \rightarrow 0$. 
(3) Here the only issue is keeping straight where everything is: we have

$$
M_{C}(X) \subset M(X) \subset \mathcal{L}(A, X),
$$

and for $m, n \in M_{C}(X)$ the rank-one operator $\theta_{m, n}=m n^{*}$ is therefore an element of $\mathcal{L}(X)=M(\mathcal{K}(X))$. We need to know that $m n^{*}$ is a $C$-multiplier of $\mathcal{K}(X)$. So, take $c \in C$. Since $m \in M_{C}(X)$ we have

$$
\phi_{C}(c) m \in X=\mathcal{K}(A, X)
$$

so

$$
\phi_{C}(c) m n^{*} \in \mathcal{K}(X) .
$$

Similarly, $\phi_{C}\left(c^{*}\right) n \in \mathcal{K}(X, A)$, so $n^{*} \Phi_{C}(c) \in \mathcal{K}(A, X)$, and hence

$$
m n^{*} \Phi_{C}(c) \in \mathcal{K}(X) \text {. }
$$

Thus we have shown that $m n^{*} \in M_{C}(\mathcal{K}(X))$.

(4) and (5) are similar to Lemma A.4. For example,

$$
\left(\bar{\kappa}(M(C)) \cdot M_{C}(X)\right) \cdot \kappa(C) \subset \bar{\kappa}(M(C)) \cdot X \subset X,
$$

because $C$ acts nondegenerately on $X$, while

$$
\kappa(C) \cdot\left(\bar{\kappa}(M(C)) \cdot M_{C}(X)\right) \subset \kappa(C) \cdot M_{C}(X) \subset X .
$$

Remark A.10. It will be useful to explicitly note the following special case of (3) above: taking $C=A$ and $\kappa=\operatorname{id}_{A}$ we get

$$
\mathcal{K}\left(M_{A}(X)\right) \subset M_{A}(\mathcal{K}(X)) .
$$

Note that

$$
M_{A}(X)=\left\{m \in M(X): \phi_{A}(a) m \in X \text { for all } a \in A\right\},
$$

so that, unlike $M(X)$, the set $M_{A}(X)$ depends upon the left module map $\phi_{A}$.

The following generalizes EKQR06, Proposition 1.42]:

Proposition A.11. Let $X$ and $Y$ be nondegenerate correspondences over $A$ and $B$, respectively, let $\kappa: C \rightarrow M(A)$ and $\sigma: D \rightarrow M(B)$ be nondegenerate homomorphisms, let $(\psi, \pi): X \rightarrow M_{D}(Y)$ be a correspondence homomorphism, and let $\lambda: C \rightarrow M(\sigma(D))$ be a nondegenerate homomorphism, such that

$$
\pi(\kappa(c) a)=\lambda(c) \pi(a) \quad \text { for } \quad c \in C, a \in A .
$$

Then $(\psi, \pi)$ extends uniquely to a $C$-strict to $D$-strictly continuous correspondence homomorphism $(\bar{\psi}, \bar{\pi})$ from the $M_{C}(A)$-correspondence $M_{C}(X)$ to the $M_{D}(B)$ correspondence $M_{D}(Y)$.

Proof. We take $\bar{\pi}: M_{C}(A) \rightarrow M_{D}(B)$ as in Lemma A.5. The unique existence of $\bar{\psi}$ is proved similarly to Lemma A.5. one shows first that $\psi$ is $C$-strict to $D$ strictly continuous, so that by completeness there is a unique continuous linear extension, which must be a correspondence homomorphism by separate continuity of the operations.

Remark A.12. Again, the above proposition could be the start of an investigation of what one might call "decorated correspondences", where $C$ decorates the $A$ correspondence $X$, etc.

Here is the Toeplitz version of Proposition A.11. 
Corollary A.13. Let $X$ be a nondegenerate A-correspondence, let $\kappa: C \rightarrow M(A)$ and $\sigma: D \rightarrow M(B)$ be nondegenerate homomorphisms, let $(\psi, \pi): X \rightarrow B$ be a Toeplitz representation, and let $\lambda: C \rightarrow M(\sigma(D))$ be a nondegenerate homomorphism, such that

$$
\pi(\kappa(c) a)=\lambda(c) \pi(a) \quad \text { for } \quad c \in C, a \in A .
$$

Then $(\psi, \pi)$ extends uniquely to a $C$-strict to D-strictly continuous Toeplitz representation $(\bar{\psi}, \bar{\pi})$ from the $M_{C}(A)$-correspondence $M_{C}(X)$ to $M_{D}(B)$.

Proof. Apply Proposition A.11 with $Y=B$, regarded as a $B$-correspondence in the usual way.

Corollary A.14. Let $X$ be a nondegenerate A-correspondence, and let $(\psi, \pi)$ : $X \rightarrow B$ be a Toeplitz representation with $\pi$ nondegenerate. Then:

(1) $(\psi, \pi)$ extends uniquely to an A-strictly continuous Toeplitz representation $(\bar{\psi}, \bar{\pi})$ from the $M(A)$-correspondence $M_{A}(X)$ to $M_{A}(B)$.

(2) $\psi^{(1)}: \mathcal{K}(X) \rightarrow B$ extends uniquely to an A-strictly continuous homomorphism $\overline{\psi^{(1)}}: M_{A}(\mathcal{K}(X)) \rightarrow M_{A}(B)$.

Moreover, we have:

(a) $\overline{\psi^{(1)}}(n \cdot m)=\bar{\pi}(n) \overline{\psi^{(1)}}(m)$ and $\overline{\psi^{(1)}}(m \cdot n)=\overline{\psi^{(1)}}(m) \bar{\pi}(n)$ for all $n \in M(A)$ and $m \in M_{A}(\mathcal{K}(X))$;

(b) $\overline{\psi^{(1)}}\left(m n^{*}\right)=\bar{\psi}(m) \bar{\psi}(n)^{*}$ for all $m, n \in M_{A}(X)$.

Proof. (1) Apply Corollary A.13 with $C=D=A, \kappa=\lambda=\mathrm{id}_{A}$, and $\sigma=\pi$.

(2) First we need to justify the meaning of (b): for $m, n \in M_{A}(X)$ we need to know that $m n^{*} \in M_{A}(\mathcal{K}(X))$. But this follows easily from work we have already done: since $X$ is a $\mathcal{K}(X)-B$ imprimitivity bimodule, arguments similar to those in the proof of Lemma A.9 (2) show that the left-hand inner product on $M_{A}(X)$ takes values in $M_{A}(\mathcal{K}(X))$.

To prove (2), we apply Corollary A.5 with $C, A, B, D$ replaced by $A, \mathcal{K}(X), B, A$, respectively; recall that for $a \in A$ and $T \in \mathcal{K}(X)$ we have

$$
\psi^{(1)}(\varphi(a) T)=\pi(a) \psi^{(1)}(T) .
$$

Now everything in (2) except for (b) follows from Corollary A.5. Then (b) follows from separate continuity of the operations, density of $A, X$, and $\mathcal{K}(X)$ in $M(A)$, $M_{A}(X)$, and $M_{A}(\mathcal{K}(X))$, respectively, and the corresponding property of $\psi^{(1)}$.

Example A.15. We should justify our need for (2) in above corollary: if $\left(k_{X}, k_{A}\right)$ is the canonical Toeplitz representation of the correspondence $X=\mathcal{H}(E)$ associated to a topological graph $E$, then $k_{X}^{(1)}: \mathcal{K}(X) \rightarrow C^{*}(E)$ can be degenerate. For example, let $E$ be the directed graph in Example A.1. Then

$$
k_{X}^{(1)}(\mathcal{K}(X))=\left(\begin{array}{cc}
* & 0 \\
0 & 0
\end{array}\right),
$$

so

$$
k_{X}^{(1)}(\mathcal{K}(X)) C^{*}(E)=\left(\begin{array}{ccc}
* & 0 \\
0 & 0
\end{array}\right) M_{2}=\left(\begin{array}{cc}
* * \\
0 & 0
\end{array}\right),
$$

and so $k_{X}^{(1)}$ is degenerate.

Remark A.16. Although we will have no use for it in the main body of this paper, we point out that all of the above can be done with an "asymmetric" version of the $C$-multiplier bimodules; namely allowing correspondences with different left and 
right coefficient algebras. It takes no extra effort to establish the more general concepts and results. Since we feel that they may be useful elsewhere, we record here the asymmetric versions:

Definition A.17. Let $X$ be a nondegenerate $A-B$ correspondence, and let $\kappa_{C}$ : $C \rightarrow M(A)$ and $\kappa_{D}: D \rightarrow M(B)$ be nondegenerate homomorphisms.

(1) A $C, D$-multiplier of $X$ is a multiplier $m \in M(X)$ such that

$$
\kappa_{C}(C) \cdot m \cup m \cdot \kappa_{D}(D) \subset X,
$$

and $M_{C, D}(X)$ denotes the set of all $C, D$-multipliers of $X$.

(2) The $C, D$-strict topology on $M_{C, D}(X)$ is generated by the seminorms

$$
m \mapsto\left\|\kappa_{C}(c) \cdot m\right\| \quad \text { and } \quad m \mapsto\left\|m \cdot \kappa_{D}(d)\right\| \quad \text { for } \quad c \in C, d \in D \text {. }
$$

\section{Lemma A.18.}

(1) The $C, D$-strict topology is stronger than the relative strict topology on $M_{C, D}(X)$.

(2) $M_{C, D}(X)$ is an $M_{C}(A)-M_{D}(B)$ correspondence with respect to the restrictions of the operations of the $M(A)-M(B)$ correspondence $M(X)$, and the operations are separately continuous for the $C, D$-strict, $C$-strict, and D-strict topologies.

(3) $M_{C, D}(X)$ is the $C, D$-strict completion of $X$.

(4) $M_{C, D}(X)$ is an $M(C)-M(D)$ subbimodule of $M(X)$.

Proposition A.19. Let $X$ and $Y$ be nondegenerate $A-B$ and $P-Q$ correspondences, respectively, let $\kappa_{C}: C \rightarrow M(A), \kappa_{D}: D \rightarrow M(B), \kappa_{R}: R \rightarrow$ $M(P)$, and $\kappa_{S}: S \rightarrow M(Q)$ be nondegenerate homomorphisms, let ${ }_{\pi} \psi_{\theta}:{ }_{A} X_{B} \rightarrow$ $M_{R}(P) M_{R, S}(Y)_{M_{S}(Q)}$ be a correspondence homomorphism, and let $\lambda_{C}: C \rightarrow M\left(\kappa_{R}(R)\right)$ and $\lambda_{D}: D \rightarrow M\left(\kappa_{S}(S)\right)$ be nondegenerate homomorphisms, such that for $c \in C$, $a \in A, b \in B$, and $d \in D$ we have

$$
\pi\left(\kappa_{C}(c) a\right)=\lambda_{C}(c) \pi(a) \quad \text { and } \quad \theta\left(\kappa_{D}(d) b\right)=\lambda_{D}(d) \theta(b) .
$$

Then ${ }_{\pi} \psi_{\theta}$ extends uniquely to a $C, D$-strict to $R, S$-strictly continuous correspondence homomorphism $\bar{\pi} \bar{\psi}_{\bar{\theta}}$ from the $M_{C}(A)-M_{D}(B)$ correspondence $M_{C, D}(X)$ to the $M_{R}(P)-M_{S}(Q)$ correspondence $M_{R, S}(Y)$.

\section{REFERENCES}

[ADR00] C. Anantharaman-Delaroche and J. Renault, Amenable groupoids, Monographies de L'Enseignement Mathématique [Monographs of L'Enseignement Mathématique], vol. 36, L'Enseignement Mathématique, Geneva, 2000, With a foreword by Georges Skandalis and Appendix B by E. Germain.

[Bau93] G. Baumslag, Topics in combinatorial group theory, Lectures in Mathematics ETH Zürich, Birkhäuser Verlag, Basel, 1993.

[BH99] M. R. Bridson, A. Haefliger, Metric spaces of non-positive curvature, Grundlehren der Mathematischen Wissenschaften, 319. Springer-Verlag, Berlin, 1999.

[BO08] N. P. Brown, N. Ozawa, $C^{*}$-algebras and finite-dimensional approximations, Graduate Studies in Mathematics, 88. American Mathematical Society, Providence, RI, 2008.

[EKQR06] S. Echterhoff, S. Kaliszewski, J. Quigg, and I. Raeburn, A Categorical Approach to Imprimitivity Theorems for $C^{*}$-Dynamical Systems, vol. 180, Mem. Amer. Math. Soc., no. 850, American Mathematical Society, Providence, RI, 2006.

[FLR00] N. J. Fowler, M. Laca, I. Raeburn, The $C^{*}$-algebras of infinite graphs, Proc. Amer. Math. Soc. 128 (2000), 2319-2327. 
[GT01] J. L. Gross and T. W. Tucker, Topological graph theory, Dover Publications Inc., Mineola, NY, 2001, Reprint of the 1987 original [Wiley, New York] with a new preface and supplementary bibliography.

[HN08] G. Hao and C.-K. Ng, Crossed products of $C^{*}$-correspondences by amenable group actions, J. Math. Anal. Appl. 345 (2008), no. 2, 702-707.

[HRW05] A. an Huef, I. Raeburn, and D. P. Williams, A symmetric imprimitivity theorem for commuting proper actions, Canad. J. Math. 57 (2005), 983-1011.

[Hus94] D. Husemoller, Fibre bundles, third ed., Graduate Texts in Mathematics, vol. 20, Springer-Verlag, New York, 1994.

[KQR97] S. Kaliszewski, J. Quigg, and I. Raeburn, Duality of restriction and induction for $C^{*}$-coactions, Trans. Amer. Math. Soc. 349 (1997), 2085-2113.

[KQR08] _ Proper actions, fixed-point algebras and naturality in nonabelian duality, J. Funct. Anal. 254 (2008), 2949-2968.

[KQR10] , Skew products and coactions for topological graphs, preprint, 2010.

[Kas88] G. G. Kasparov, Equivariant $K K$-theory and the Novikov conjecture, Invent. Math. 91 (1988), no. 1, 147-201.

[Kat02] T. Katsura, Continuous graphs and crossed products of Cuntz algebras, Sūrikaisekikenkyūsho Kōkyūroku (2002), no. 1291, 73-83, Recent aspects of $C^{*}$ algebras (Japanese) (Kyoto, 2002).

[Kat04] - A class of $C^{*}$-algebras generalizing both graph algebras and homeomorphism $C^{*}$-algebras. I. Fundamental results, Trans. Amer. Math. Soc. 356 (2004), no. 11, $4287-4322$.

[Kat06] $\quad$ A class of $C^{*}$-algebras generalizing both graph algebras and homeomorphism $C^{*}$-algebras. III. Ideal structures, Ergodic Theory Dynam. Systems 26 (2006), no. 6, $1805-1854$.

[Kat08] $\quad$ A class of $C^{*}$-algebras generalizing both graph algebras and homeomorphism $C^{*}$-algebras. IV. Pure infiniteness, J. Funct. Anal. 254 (2008), no. 5, 1161-1187.

[KK97] A. Kishimoto and A. Kumjian, Crossed products of Cuntz algebras by quasi-free automorphisms, Operator algebras and their applications (Waterloo, ON, 1994/1995), Fields Inst. Commun., vol. 13, Amer. Math. Soc., Providence, RI, 1997, pp. 173-192.

[KP99] A. Kumjian and D. Pask, $C^{*}$-algebras of directed graphs and group actions, Ergod. Th. and Dynam. Sys. 19 (1999), 1503-1519.

[LS77] R. C. Lyndon, P. E. Schupp, Combinatorial group theory, Ergebnisse der Mathematik und ihrer Grenzgebiete, Band 89. Springer-Verlag, Berlin-New York, 1977.

[Mas91] W. S. Massey, A basic course in algebraic topology, Graduate Texts in Mathematics, vol. 127, Springer-Verlag, New York, 1991.

[OP78] D. Olesen and G. K. Pedersen, Applications of the Connes spectrum to $C^{*}$-dynamical systems, J. Funct. Anal. 30 (1978), no. 2, 179-197.

[OP80] - Applications of the Connes spectrum to $C^{*}$-dynamical systems. II, J. Funct. Anal. 36 (1980), no. 1, 18-32.

[Pal61] R. S. Palais, On the existence of slices for actions of non-compact Lie groups, Ann. of Math. (2) 73 (1961), 295-323.

[QR95] J. C. Quigg and I. Raeburn, Induced $C^{*}$-algebras and Landstad duality for twisted coactions, Trans. Amer. Math. Soc. 347 (1995), 2885-2915.

[Qui92] J. C. Quigg, Landstad duality for $C^{*}$-coactions, Math. Scand. 71 (1992), 277-294.

[Rae05] I. Raeburn, Graph algebras, CBMS Regional Conference Series in Mathematics, vol. 103, Published for the Conference Board of the Mathematical Sciences, Washington, DC, 2005.

[Rie90] M. A. Rieffel, Proper actions of groups on $C^{*}$-algebras, Mappings of operator algebras (Philadelphia, PA, 1988) (Boston, MA), Birkhäuser Boston, 1990.

[Rie04] - Integrable and proper actions on $C^{*}$-algebras, and square-integrable representations of groups, Expo. Math. 22 (2004), 1-53.

[RW98] I. Raeburn and D. P. Williams, Morita equivalence and continuous-trace $C^{*}$-algebras, Math. Surveys and Monographs, vol. 60, American Mathematical Society, Providence, RI, 1998. 
Department of Mathematics, University of Nevada, Reno NV 89557-0084, USA

E-mail address, Valentin Deaconu: vdeaconu@unr.edu

Department of Mathematics, University of Nevada, Reno NV 89557-0084, USA

E-mail address, Alex Kumjian: alex@unr.edu

School of Mathematical and Statistical Sciences, Arizona State University, Tempe AZ 85287-1804, USA

E-mail address, John Quigg: quigg@asu.edu 